\title{
Quién es quién en la red de coautoría en Colombia*
}

\author{
Recibido: 21 de enero de 2021 - Aceptado: 20 de abril de 2021
}

Doi: https://doi.org/10.12804/revistas.urosario.edu.co/economia/a.10471

\author{
Carlos León ${ }^{\dagger}$ \\ Angélica Bahos-Olivera ${ }^{\ddagger}$
}

\section{Resumen}

En esta investigación se estudia la red de coautoría de artículos publicados entre el 2010 y el 2019 en revistas indexadas en Scopus, del área de economía, econometría y finanzas, en las que alguno de los autores está afiliado a una institución en Colombia. Conseguimos visualizar y describir la estructura conectiva de la red de coautoría. Pese a que la red es poco interconectada, existen unos cuantos autores altamente vinculados que la mantienen unida y con una corta distancia entre los investigadores que la conforman, en lo que se conoce como una red de mundo pequeño. Luego de descartar a autores ocasionales, se cuantificó la importancia de estos por su contribución a la red de coautoría. Es de resaltar la importancia de los autores afiliados al Banco de la República (el banco central de Colombia), quienes contribuyen de gran manera a la estructura conectiva de la red. Este es un primer paso hacia el estudio de la red de publicaciones indexadas del área de economía en el caso colombiano.

* Las opiniones contenidas en este documento son responsabilidad exclusiva de los autores y no comprometen al Banco de la República ni a su Junta Directiva. Agradecemos a Freddy Cepeda, Mauricio Villamizar, Humbert Garrido y a dos evaluadores anónimos, por sus comentarios y sugerencias. Cualquier error es de los autores.

+ Departamento de Seguimiento a la Infraestructura Financiera, Banco de la República, Bogotá, Colombia; Departamento de Finanzas, Universidad de Tilburg, Tilburg, Países Bajos. Email: cleonrin@banrep.gov.co, c.e.leonrincon@tilburguniversity.edu oRcID: https://orcid.org/0000-0001-5357-0216

‡ Centro de Apoyo a la Investigación Económica, Banco de la República, Bogotá, Colombia. Email: cbahosol@banrep.gov.co orcID: https://orcid.org/0000-0003-1474-9023

Para citar este artículo: León, C., \& Bahos-Olivera, A. (2021). Quién es quién en la red de coautoría en Colombia. Revista de Economía del Rosario, 24(2), 1-39. https://doi.org/10.12804/ revistas.urosario.edu.co/economia/a.10471 
Palabras clave: redes; coautoría; investigación; centralidad.

Clasificación JEL: L14, A14, D85, O30.

\title{
Who's Who in the Colombian Co-Authorship Network?
}

\begin{abstract}
We study the co-authorship network of articles published between 2010 and 2019 in Scopus-indexed journals in the economics, econometrics, and finance subject areas with at least one of the authors affiliated to a Colombian institution. We visualize and describe the connective structure of the co-authorship network. Although it is sparsely connected, there are a few well-connected authors that keep it together and allow others to be close to each other, which is commonly known as a small-world network; this concurs with related literature. After discarding occasional authors, we quantify the importance of authors as contributors to the network. Results show the importance of authors affiliated to the Banco de la República (Colombia's central bank), who decisively contribute to the connective structure of the network. This article is a first step towards the study of indexed publications in economics in the Colombian case.
\end{abstract}

Keywords: Networks; coauthorship; research; centrality. JEL classification: L14, A14, D85, O30.

\section{Quem é quem na rede de coautoria na Colômbia}

\section{Resumo}

Neste artigo estudamos a rede de coautorias de artigos publicados entre 2010 e 2019 em revistas indexadas no Scopus, na área de economia, econometria e finanças, onde algum dos autores estivesse filiado a uma instituição na Colômbia. Conseguimos visualizar e descrever a estrutura conectiva da rede de coautoria. Apesar de a rede não ser muito interconectada, existem alguns autores altamente interconectados que a mantêm unida e a uma curta distância entre os autores que a compõem, no que se conhece como uma rede de um mundo pequeno. Após descartar autores ocasionais, quantificamos a importância dos autores em sua contribuição para a rede de coautoria. Destaca-se a importância dos autores filiados ao Banco da República (o banco central da Colômbia), os quais contribuem sobremaneira para a estrutura conectiva da rede. Este é um primeiro passo para o estudo da rede de publicações indexadas na área de economia no caso colombiano.

Palavras-chave: redes; coautoria; pesquisa; centralidade.

Classificação JEL: L14, A14, D85, O30. 


\section{Introducción}

La red de coautoría es una representación del sistema que emerge de las relaciones colaborativas existentes entre autores que investigan en un área del conocimiento. Su estudio es importante por varias razones. La red de coautoría refleja la influencia intelectual y social entre investigadores (Katz \& Martin, 1997), y es la unidad crítica de producción de conocimiento (Melin \& Persson, 1996). Esta red no solo representa una sociedad académica, sino que permite describir la estructura de la generación de conocimiento sobre un tema o área en particular (Newman, 2001). Son las redes sociales de mayor tamaño que se disponen de manera pública (Kumar, 2015). Y, dado que la colaboración es un proceso social dinámico, el resultado de los estudios de la red de coautoría puede inducir cambios en el comportamiento de los autores e instituciones relacionadas con el proceso de investigación (Katz \& Martin, 1997; Kumar, 2015).

En este artículo se estudió la red de coautoría de artículos publicados en revistas indexadas en Scopus ${ }^{1}$ entre el 2010 y el 2019, del área de economía, econometría y finanzas, en la cual alguno de los autores está afiliado a una institución en Colombia. Con esta investigación se consiguió visualizar y describir la estructura conectiva de la red de coautoría, así como cuantificar la importancia de los autores por su contribución a la producción conjunta de artículos de investigación.

El estudio de las redes de coautoría tiene varios usos prácticos (Uddin et al., 2013; Kumar, 2015). Primero, permite a los investigadores identificar potenciales pares con los cuales colaborar en un tema particular. Segundo, facilita descubrir el grado de integración en el proceso de generación de conocimiento, así como el flujo de dicho conocimiento. Tercero, con base en la importancia de los autores en la red de coautoría, es posible establecer una clasificación o ranking de los investigadores que participan de ella; como señalan Kumar (2015) y Hsieh et al. (2018), este tipo de clasificación —basada en la importancia en la red de coautoría- puede ser útil para optimizar la asignación de recursos para la investigación. Cuarto, en unión con otras bases de datos - e.g., citas bibliográficas de los artículos, factor de impacto de las revistas - o características de los autores - e.g., género, edad, afiliación-, es posible estudiar otros aspectos relevantes de la colaboración entre investigadores. En este caso, nos concentramos en los tres primeros usos

1 Como se presenta en una sección posterior, Scopus es la mayor base de datos multidisciplinar de citas y resúmenes bibliográficos de publicaciones evaluadas por pares, que brinda acceso a cerca de 39.500 piezas de investigación. 
prácticos; para el cuarto uso, se dejan algunas bases para futuros trabajos de investigación.

Esta investigación contribuye a la literatura sobre redes de coautoría. La mayor parte de esta literatura se concentra en áreas del conocimiento diferentes a la economía, como la medicina, las matemáticas y la biología. El presente trabajo se une a los estudios realizados sobre redes de coautoría en el área de economía por Goyal et al. (2006), Fatt et al. (2010), ŁopaciukGonczaryk et al. (2015), Larrosa (2019) y Tabak et al. (2021).

Así mismo, este estudio complementa el trabajo realizado por el convenio Red de Investigadores de Economía. ${ }^{2}$ Como parte de sus actividades, ese convenio publica la red de coautorías y el Top 100 de autores colombianos según la base de datos bibliográfica de RePEc (Research Papers in Economics). ${ }^{3}$ Sin embargo, dado que la base bibliográfica de Scopus se limita a un subconjunto de revistas indexadas, en la cual los artículos son publicados luego de la evaluación por parte de pares académicos, la red de coautoría y la clasificación que resultan de nuestro estudio pueden considerarse como versiones depuradas y complementarias de aquellas obtenidas con base en RePEc. ${ }^{4}$

En lo que sigue nos referiremos indistintamente a coautoría y colaboración, a investigadores y autores, a artículos de investigación y publicaciones, a revistas indexadas en Scopus y revistas, y a la clasificación "economía, econometría y finanzas" de Scopus y a economía. Además de esta introducción, el documento se compone de las siguientes secciones. La segunda sección presenta una revisión de la literatura sobre coautoría en el área de economía. La tercera describe la base de datos, así como el procedimiento desarrollado para su construcción y depuración. La cuarta expone el análisis de redes como metodología para estudiar la red de coautoría. La quinta muestra los resultados en dos subsecciones que corresponden a la estructura conectiva

2 La Red de Investigadores de Economía es un convenio entre 22 universidades colombianas y el Banco de la República (i.e., el banco central de Colombia) para ampliar la difusión de investigaciones académicas en distintas áreas de economía. Véase: https:// www.redinvestigadores.org/about

3 Véase Ideas: https://ideas.repec.org/top/top.colombia.html

4 Al igual que Google Scholar (https://scholar.google.com), RePEc es una base de datos que no se limita a publicaciones evaluadas por pares académicos. De acuerdo con Martin-Martin et al. (2018), si bien Google Scholar ofrece una base de datos más amplia, el impacto académico de los documentos que sí tiene Google Scholar (y que no están en Scopus) es más bajo. Dada la semejanza entre RePEc y Google Scholar, utilizar la base de datos de Scopus resulta en un estudio que se concentra en el subconjunto de aquellos documentos de mayor impacto académico, por tanto, es un estudio depurado frente a utilizar la base de datos de RePEc. 
de la red de coautoría y a la contribución de los coautores a la red. La última sección concluye y sugiere algunas alternativas de investigación futura.

\section{La coautoría en economía}

La coautoría de artículos de investigación es un indicador parcial pero conveniente de la colaboración entre investigadores (Melin \& Persson, 1996; Katz \& Martin, 1997; Uddin et al., 2013; Kumar, 2015). Es parcial porque es difícil establecer la verdadera contribución de los autores ${ }^{5}$ y porque no toda colaboración conduce a una publicación. Es conveniente por la disponibilidad de una gran cantidad y calidad de datos sobre la red social que forman los investigadores voluntariamente, con lo cual su uso permite mediciones que son pertinentes, objetivas, verificables, reproducibles y de bajo costo.

Al igual que otras áreas del conocimiento, ${ }^{6}$ la investigación en economía se caracteriza cada vez más por la coautoría (Hollis, 2001; Rath \& Wohlrabe, 2016; Larrosa, 2019; Hamermesh, 2020; O’Hagan \& Kuld, 2020; Essers et al., 2020). Según Hamermesh (2020), la gran mayoría de artículos académicos en el área de economía tiene dos o más autores, y una gran parte tiene tres o más. O’Hagan y Kuld (2020) documentan que la proporción de artículos en revistas del área de economía con un solo autor pasó de la mitad en 1996 a la cuarta parte en el 2014; entre tanto, la proporción de aquellos publicados por tres coautores pasó de cerca de una décima a una cuarta parte.

Existen diversas hipótesis —no excluyentes- que buscan explicar el aumento en la coautoría (Katz \& Martin, 1997; Hollis, 2001; Lu et al., 2019; O’Hagan \& Kuld, 2020). La primera tiene que ver con la división del trabajo, según la cual, la coautoría permite aprovechar la especialización de los autores para enfrentar la mayor exigencia e interdisciplinariedad del mundo académico. ${ }^{7}$ La segunda corresponde a la necesidad de compensar la ayuda prestada por otros investigadores — quienes no se conformarán con un agradecimiento- - La tercera se relaciona con la aversión al riesgo de los investigadores, quienes enfrentan la creciente presión por publicar (i.e., publish or perish) produciendo varios artículos de manera conjunta; en ese sentido, la

5 Por ejemplo, hay evidencia de la inclusión de falsos autores por razones sociales o estratégicas y, de denegación de coautoría cuando existe distancia social entre los autores (e.g., profesor-alumno) (Melin \& Persson, 1996; Katz \& Martin, 1997; Uddin et al., 2013).

6 Para una revisión de la literatura sobre redes de coautoría en diferentes áreas del conocimiento, véase Kumar (2015).

$7 \quad$ El estudio de Tabak et al. (2021) sobre coautoría en el tema de redes del mercado interbancario brinda evidencia sobre la importancia de la interdisciplinariedad en la colaboración académica en el área de economía. 
coautoría no solo permite aumentar el número de artículos publicados por autor, sino que también diversificar el riesgo de fracaso de los proyectos de investigación. La cuarta es respecto a la posibilidad de aumentar la popularidad, visibilidad y reconocimiento de los artículos, lo cual tiene un impacto en la reputación y evaluación de los autores. ${ }^{8}$ La quinta trata de los avances y menores costos en comunicación y transporte, los cuales han facilitado la colaboración entre investigadores.

No existe consenso sobre el impacto de las relaciones de coautoría en la calidad y cantidad de la investigación. Hollis (2001) encuentra que coexisten efectos positivos y negativos de la coautoría, los primeros consisten en una mayor calidad, extensión y número de artículos publicados, mientras que los negativos consisten en una menor producción que puede ser atribuible a cada coautor. ${ }^{9}$ No obstante, existen efectos que no son medidos y que pueden compensar los negativos, como son el aprendizaje y transmisión de conocimiento, la satisfacción del trabajo con amigos o colegas, la diversificación del riesgo de fracaso de los proyectos de investigación y, la mayor influencia y renombre que tienen los trabajos en coautoría (Katz \& Martin, 1997; Hollis, 2001). Adicionalmente, dado que en la academia no es usual que se reparta la producción por coautor para evaluar su desempeño (Liebowitz, 2020), la coautoría puede resultar eficiente para los investigadores, aunque puede inducir a la inclusión de falsos autores.

Pese a que la publicación en conjunto es una práctica que lleva varios siglos, el inicio del estudio de las redes de coautoría data de la década de los noventa, con un impulso reciente gracias a la disponibilidad de grandes bases de datos bibliográficas (Uddin et al., 2013; Kumar, 2015). ${ }^{10}$ En su mayoría, el análisis de redes (Albert \& Barabási, 2002; Börner et al., 2007; Newman, 2010) es la base metodológica de esos estudios. Así, los autores son los elementos o nodos en las redes y las relaciones de coautoría, se representan como

8 Existe evidencia que señala que los artículos en coautoría tienden a ser más influyentes y otorgan mayor renombre a los autores (Katz \& Martin, 1997). Así mismo, adicionar un autor a un artículo tiene un efecto positivo pero marginal en el número de citas de este (Hamermesh, 2020); empero, si se ajustan las citas de los artículos por autor, ese efecto es negativo ( $\mathrm{O}^{\prime}$ Hagan \& Kuld, 2020).

9 Según Hollis (2001), adicionar un autor se relaciona con una reducción en la producción per cápita de entre 7 y $20 \%$.

10 Quizá el caso de coautoría más popular es aquel que involucra al matemático húngaro Paul Erdös (1913-1996), a quien se le atribuyen más de 1.500 artículos con más de 500 coautores. En este caso, los autores buscan establecer a cuántas coautorías de distancia se encuentran de Erdös, en lo que se conoce como el número de Erdös. Cualquier coautor de Erdös tiene una distancia de 1; cualquier coautor de un coautor de Erdös (sin ser coautor de Erdös) tiene una distancia de 2, y así sucesivamente (Newman, 2001; Barabási et al., 2002; Uddin et al., 2013). 
conexiones o relaciones entre los nodos. Se utilizan diversas medidas para describir la estructura conectiva de las redes de coautoría y para cuantificar la importancia de los autores en estas.

Respecto a la estructura conectiva y la importancia de los autores, los principales hallazgos con redes de coautoría (Newman, 2001; Barabási et al., 2002; Albert \& Barabási, 2002; Newman, 2004; Liu et al., 2005; Goyal et al., 2006; Yan \& Ding, 2009; Kumar, 2015; Larrosa, 2019; Essers et al., 2020) coinciden con aquellos de muchas otras redes estudiadas en la literatura (Albert \& Barabási, 2002; Barabási et al., 2002; Newman, 2010). En general, son redes en las cuales el número de coautorías observadas con respecto al número de coautorías posibles es bajo, en las que los autores no se encuentran alejados entre sí pese a su gran número; redes en las que la proporción de relaciones transitivas es importante; la distribución de las relaciones es heterogénea, con unos pocos autores con muchas relaciones; y en las cuales los autores tienden a relacionarse más con aquellos que le sean semejantes por el número de coautorías.

Existen algunos estudios de redes de coautoría en el área de economía. Goyal et al. (2006) estudian la red de coautorías con base en las revistas incluidas en EconLit entre 1979 y 1999. Fatt et al. (2010) analizan la red de coautorías que resulta de los artículos publicados en el Journal of Finance entre 1980 y el 2009. Łopaciuk-Gonczaryk et al. (2015) examinan las relaciones de coautoría producto de las publicaciones de autores polacos en cinco revistas líderes en Polonia entre 1999 y el 2012. Larrosa (2019) revisa la red de coautoría que resulta de la participación en la Reunión Anual de la Asociación de Economía Política de Argentina entre 1964 y el 2014. Essers et al. (2020) indagan la red de coautoría de los Working Papers del Fondo Monetario Internacional entre 1990 y el 2017. Tabak et al. (2021) investigan la red de coautoría de las publicaciones en Scopus y Web of Science relacionadas con el tema de redes del mercado interbancario. Cárdenas-Tapia (2021) estudia 11 redes de coautoría de varias disciplinas en México, entre el 2018 y el 2020, incluida economía. En relación con nuestro objetivo de investigación, en estos casos se reporta que la estructura conectiva de las redes de coautoría en el área de economía coincide con aquella de otras áreas y de redes de otros tipos.

\section{Base de datos}

La construcción y estudio de redes de coautoría es posible gracias al desarrollo de bases de datos especializadas que recolectan, procesan, consolidan y distribuyen la información sobre las publicaciones. En el área de economía, existen varias bases de datos ampliamente utilizadas: Scopus, Web of 
Science, RePEc y Google Scholar. Cada una de ellas cuenta con una cobertura diferente respecto a los documentos que indexa, con ventajas y desventajas frente al uso que se quiera hacer de estas. ${ }^{11}$ Así mismo, existen alternativas al uso de bases de datos especializadas, como son los documentos presentados en conferencias (Larrosa, 2019), documentos publicados en una o varias revistas de renombre (Fatt et al., 2010; Łopaciuk-Gonczaryk et al., 2015) o al interior de una institución (Essers et al., 2020).

De estas alternativas, seleccionamos la base de datos Scopus. Scopus es la mayor base de datos multidisciplinar de citas y resúmenes bibliográficos de publicaciones evaluadas por pares, que brinda acceso a cerca de 39.500 piezas de investigación en diferentes áreas del conocimiento. Las revistas que son indexadas en Scopus han superado requisitos de políticas de publicación, calidad del contenido, cumplimiento de la periodicidad declarada y, análisis de citas. A diferencia de RePEc y Google Scholar, Scopus y Web of Science realizan un proceso de evaluación para admitir las revistas - las cuales se conocen como "indexadas"- Al comparar Scopus y Web of Science en el área de economía, el índice de Scopus cuenta con cerca de 1.100 revistas, mientras que Web of Science cuenta con cerca de 370. Adicionalmente, Scopus cuenta con una mayor cobertura en Ciencias Sociales y documentos escritos en español.

Para este análisis, se extrajo la base de datos de artículos publicados en el área de economía entre el 2010 y el 2019, en los que por lo menos uno de los autores está afiliado a una institución en Colombia. ${ }^{12}$ Se eligió este periodo de diez años por ser lo suficientemente extenso como para obtener una caracterización estable, pero vigente de las relaciones de coautoría. Un periodo más corto podría solo capturar las relaciones de coautoría recientes, mientras que un periodo más largo podría contener información que ya no es pertinente para caracterizar las relaciones de coautoría en la actualidad. Así como en Goyal et al. (2006), no se consideran documentos de trabajo ni libros - solo artículos publicados en revistas indexadas-.

11 En el Apéndice B se describen y comparan las bases RePEc, Scopus, Web of Science y Google Scholar.

12 La ecuación de búsqueda en Scopus es la siguiente: AfFilcountry (colombia ) AND ( LiMit-To ( DOCTYPe, "ar") ) AND ( LIMIT-To ( SUbJAREA, "ECON") ) AND ( LIMIT-TO ( PUBYEAR , 2019 ) OR LIMIT-TO ( PUBYEAR, 2018 ) OR LIMIT-TO ( PUBYEAR, 2017 ) OR LIMITTO ( PUBYEAR , 2016 ) OR LIMIT-TO ( PUBYEAR , 2015 ) OR LIMIT-TO ( PUBYEAR, 2014 ) OR LIMIT-TO ( PUBYEAR, 2013 ) OR LIMIT-TO ( PUBYEAR, 2012 ) OR LIMIT-TO ( PUBYEAR, 2011 ) OR LIMIT-To ( PUBYEAR, 2010 ) ). Como resultado de la búsqueda, se obtuvo una base de datos de 2.401 artículos, publicados en 426 revistas, escritos por 4.124 autores (antes del proceso de normalización descrito a continuación). 
Al igual que Newman (2001) y Goyal et al. (2006), distinguimos a los autores por su apellido e iniciales. Sin embargo, ante la falta de normalización con la que los autores firman sus artículos, se realizó una exhaustiva revisión para desambiguar varios casos, entre ellos: en los que dos o más autores compartían apellidos e iniciales, esclarecer autorías en la que se ha recuperado tan solo el segundo apellido de los autores, unificar autores con diferencias en su apellido e iniciales debidas al uso (o no) de acentos, agrupar autores que usan de forma variable uno o dos apellidos y uno o dos nombres, así como aquellos en los cuales el uso de preposiciones como "de" o "del" generaban una incorrecta indexación. A cada autor se le agregó su afiliación, la cual se determinó con base en aquella reportada en el artículo más reciente en la base de datos. El género de cada autor se determinó de acuerdo a su nombre, en algunos casos se verificó en los perfiles de los autores en redes sociales o en las instituciones a las que se encuentran afiliados.

\section{Metodología}

La ciencia de redes es un área de investigación altamente interdisciplinaria que tiene como objetivo mejorar nuestro entendimiento de los sistemas que nos rodean - e.g., sociales, físicos, biológicos, tecnológicos- De manera general, la ciencia de redes se divide en dos áreas principales, análisis de redes y modelado de redes (Börner et al., 2007). Con el análisis de redes se busca describir y explicar un sistema dado. Con el modelado de redes se busca diseñar procesos capaces de reproducir las redes observadas y hacer predicciones sobre ellas.

En este caso utilizaremos el análisis de redes. Con el análisis de redes se busca describir y explicar la estructura conectiva del sistema que resulta de las coautorías en publicaciones indexadas en Scopus para el conjunto de años y autores antes definidos. Para tal fin nos concentramos en dos tipos de análisis. El primero tiene que ver con la estructura conectiva de la red y el segundo con la importancia de los autores en la red.

\section{La estructura conectiva de la red}

De manera general, una red es una representación de los elementos de un sistema y de las interacciones que existen entre ellos. En el caso de una red de coautoría, los elementos son los autores y las interacciones son el vínculo de colaboración que existe al participar como coautores de un artículo. Si $i$ y $j$ son autores, una representación formal de una red de coautoría es la matriz $A$, en la que sus elementos son $a_{i j}$ : 


$$
a_{i j}=\left\{\begin{array}{l}
1 \text { si } i \text { y } j \text { publicaron un documento juntos, } \\
0 \text { en los demás casos }
\end{array}\right\}
$$

En este caso, si $N$ es el número de autores en la red, $A$ es una matriz cuadrada de dimensiones $N \times N$ que contiene la información correspondiente a la existencia o ausencia de una relación de colaboración entre los diferentes autores que participan de la red. Dado que las relaciones de coautoría son por definición recíprocas $\left(a_{i j}=a_{j i}\right), A$ es una matriz simétrica con respecto a la diagonal principal; es decir, se trata de una red no dirigida.

Además, es posible incorporar el número de publicaciones en coautoría. En ese caso, la matriz $\tilde{A}$ contiene la información correspondiente al número de trabajos en colaboración entre los diferentes autores de la red. Si se representa el número de trabajos en coautoría entre los autores $i$ y $j$ con $y=0,1,2,3 \cdots$

$$
\tilde{a}_{i j}=\{y \text { es el número de documentos que } i \text { y } j \text { publicaron juntos }\}
$$

Entonces, $A$ y $\tilde{A}$ son representaciones de la red de coautoría. Ambas son cuadradas y simétricas, pero $A$ es binaria y $\tilde{A}$ es ponderada. En la visualización de ambas matrices, los autores aparecerán como nodos o vértices, los cuales estarán interconectados por líneas que representan las relaciones de coautoría. En el caso de la representación gráfica de $A$, las líneas tendrán el mismo ancho, mientras que en la representación gráfica de $\tilde{A}$ el ancho corresponderá con el valor de $y$.

Con base en $A$ es posible calcular algunas estadísticas básicas que describen la estructura conectiva de la red de coautoría. Las estadísticas más relevantes de la red, en este caso, son la densidad; los primeros cuatro momentos de la distribución de las relaciones - i.e., media, desviación estándar, curtosis y sesgo-; el coeficiente de ley de potencia de la distribución de las relaciones de coautoría; la distancia promedio entre autores; y la transitividad y asortatividad de las relaciones de coautoría. ${ }^{13}$

En el primer caso, la densidad es una medida de qué tan interconectados están los autores. Se calcula como la fracción de relaciones de coautoría observadas con respecto a las relaciones de coautoría posibles. Una red de coautoría cuya densidad se aproxima a 1 (0) sugiere que la colaboración entre

13 El Apéndice A presenta las fórmulas para el cálculo de las estadísticas básicas de red. 
autores es muy (poco) frecuente. En el caso extremo en el que la densidad sea igual a 1 (i.e., red completa), todos los autores han colaborado entre sí; de ser igual a 0 , todas las publicaciones se hicieron de manera individual —no existe colaboración entre autores-.

En cuanto a la distribución de las relaciones en la red, los cuatro momentos de esa distribución permiten evidenciar si las relaciones se reparten de manera equitativa (i.e., homogénea) o no, entre los elementos de la red. De ser homogénea, no existirían grandes diferencias en cuanto a la contribución de los autores en la red. De acuerdo con la mayoría de las redes estudiadas en la literatura (Albert \& Barabási, 2002; Newman, 2010), la distribución de las conexiones es altamente inequitativa. La literatura sobre redes de coautoría coincide con lo anterior, lo cual sugiere que hay autores que colaboran mucho más que otros.

De manera adicional, si el coeficiente de ley de potencia de la distribución de las relaciones se aproxima al rango [2-3], no solo se valida que esa distribución de las relaciones es inequitativa, sino que la red se aproxima a lo que se conoce como redes libres de escala (scale-free) (Barabási \& Albert, 1999; Newman, 2003). ${ }^{14}$ En tal caso, no solo hay autores que colaboran mucho más que otros, sino que existe un número pequeño de autores que concentra la mayor parte de las relaciones de coautoría. En la literatura (Barabási et al., 2002; Newman, 2003; Newman, 2004) se ha documentado que muchas redes de coautoría coinciden con este tipo - aunque otras semejantes también son posibles- Como resalta Larrosa (2009), este tipo de red de coautoría es propicio para la diseminación de la información.

La distancia promedio entre autores, calculada como la media del número de relaciones de colaboración necesarias para que un autor se relacione con cualquier otro, indica qué tan cercanos son los investigadores en la red de coautoría. Por ejemplo, si los autores $i$ y $j$ tienen una relación de coautoría, su distancia es 1 ; si el autor $k$ tiene una relación de coautoría con $j$, pero no con $i$, su distancia con $i$ es de 2 - gracias a su relación con $j$ - Una red con distancia promedio baja revela que los autores son cercanos entre ellos.

14 La distribución de la ley de potencia sugiere que la probabilidad de observar un nodo con $x$ conexiones sigue la forma funcional $P x \propto z x^{\gamma}$, donde $z$ es un parámetro arbitrario y la constante $\gamma$ se conoce como el coeficiente o exponente de ley de potencia. Aquellas redes cuyas relaciones se distribuyen según la ley de potencia con $\gamma=[2,3]$, se conocen como redes libres de escala (Newman, 2003). Dado que el ajuste de ese coeficiente requiere de una gran cantidad de datos, en nuestro caso se utiliza solo cuando el número de autores así lo permite, y se usa la visualización de la distribución para propósitos de verificación. 
Esta cercanía puede resultar de una red densa o de una red en la que algunos autores sirven de buenos conectores para muchos otros.

La transitividad mide la frecuencia con que existen triángulos cerrados en la red. En el caso de las redes de coautoría, la transitividad muestra con qué frecuencia sucede que, si dos autores $i$ y $j$ tienen una relación de autoría con un autor $k$, también $i$ y $j$ están relacionados. En general, la transitividad refleja la disposición de los autores a colaborar (Barabási et al., 2002). La transitividad tiende a ser alta cuando los artículos son escritos por más de dos autores (Newman, 2001, 2004) o cuando un autor lidera un grupo que publica conjuntamente.

La asortatividad mide si existe una preferencia de los autores por conectarse con otros que tienen un número similar de coautores. Por ser calculada como una correlación, valores positivos (negativos) y cercanos a 1 (-1) muestran que existe una preferencia por publicar con coautores con un número de relaciones de coautoría similar (diferente).

Por último, es importante resaltar la topología que se conoce como red de mundo pequeño (small-world). Generalmente, se habla de redes de mundo pequeño cuando la distancia promedio entre nodos es del orden de $\ln N$ (i.e., la distancia entre nodos es reducida pese al número de nodos en la red). No obstante, Goyal et al. (2006) condicionan la existencia de una red de mundo pequeño a que la distancia promedio sea del orden $\ln N$ para una red de baja densidad y alta transitividad, con pocas (o ninguna) partes o componentes ${ }^{15}$ desconectados. Para una red de coautoría, la principal consecuencia de que su estructura conectiva sea de mundo pequeño, es la alta eficiencia para crear conocimiento a través de la publicación conjunta de artículos y del aprendizaje que supone la colaboración entre autores. Una gran cantidad de redes de autoría han sido catalogadas de mundo pequeño, donde la presencia de redes libres de escala (i.e., con autores particularmente interconectados) es importante para alcanzar la corta distancia entre coautores (Goyal et al., 2006; Kumar, 2015).

\section{La importancia de los autores en la red}

El análisis de redes también se ocupa de estudiar la contribución de los nodos a la conectividad de la red. Para tal fin se cuenta con una gran cantidad de medidas de importancia en la red, a las que se refiere como medidas de

15 Un componente en una red es un subconjunto de nodos en el que hay una relación directa o indirecta entre todos los nodos. 
centralidad. Teniendo en cuenta que la red de coautoría no es dirigida, se han seleccionado cinco medidas. ${ }^{16}$

La primera medida de importancia es la centralidad de grado (degree centrality). Esta medida corresponde al número de relaciones que tiene cada autor en la red. En el caso que una relación entre dos autores consista en más de un artículo en colaboración, solo se tomará como una única relación; por eso, se calcula con base en la matriz $A$. Como es de esperar, entre más alto el número de relaciones, más importante es el autor en la red. Autores con mayor centralidad de grado tienen una mayor habilidad para influir en otros (Larrosa, 2019). Sin embargo, es una medida que puede sesgarse fácilmente en el caso de artículos escritos por un gran número de autores (Liu et al., 2005).

La segunda medida es la centralidad de intensidad (strength centrality). Esta medida concierne a la suma del número de artículos en colaboración, publicados por cada autor en la red. A diferencia de la centralidad de grado, en este caso se tiene en cuenta la intensidad de la relación entre los autores; es decir, se calcula con base en la matriz $\tilde{A}$.

La tercera medida es la centralidad de interposición (betweenness centrality). Esta determina la importancia de un autor como intermediario entre otros autores. Para un autor $k$, se calcula con base en el número de caminos más cortos entre todas las parejas $i$ y $j$ de la red que pasa a través de $k$. Entre más caminos requieran de $k$ como conector entre los nodos, más importante es para la red. Un autor con alta centralidad de interposición es crucial para el flujo de la información en la red de coautoría (Fatt et al., 2010), y usualmente, se involucra en proyectos interdisciplinarios (Larrosa, 2019). Esta medida se calcula con base en la matriz $A$.

La cuarta medida es la centralidad de cercanía (closeness centrality). Entre más cercano sea $k$ a todos los demás autores, mayor es la posibilidad de que él o un coautor de él sea aquel quien puede ayudar a llevar un proyecto de investigación a buen término (Fatt et al., 2010; Kumar, 2015). Autores con mayor centralidad de cercanía tienen una mayor habilidad para influir a la red en su conjunto (Larrosa, 2019). Esta medida se calcula con base en la matriz $A$.

Finalmente, la quinta medida es la centralidad de eigenvector (eigenvector centrality). A diferencia de las anteriores, en esta medida la importancia de los coautores es una fuente de importancia. La centralidad de eigenvector de un

16 Estas cinco medidas son las más utilizadas en la literatura relacionada (Liu et al., 2005; Yan \& Ding, 2009; Kumar, 2015). El Apéndice A presenta las fórmulas para el cálculo de las medidas de centralidad. Todas las medidas son normalizadas para que la centralidad de cada autor corresponda a la contribución del total de los autores. 
autor $k$ resulta de la suma ponderada de la centralidad de eigenvector de todos aquellos autores con los que ha colaborado. De ese modo, la importancia de un autor no solo depende de la importancia de todos los demás autores con los que está conectado directa e indirectamente, sino también de la estructura conectiva de la red. Esta medida se puede calcular con base en ambas matrices, $A$ y $\tilde{A}$. En este caso, con el fin de reconocer la importancia de las diferencias en intensidad de las coautorías, utilizaremos la matriz $\tilde{A}$.

\section{Resultados}

Los resultados se presentan en dos partes. La primera presenta la visualización y descripción de la estructura conectiva de la red de coautorías. La segunda expone el resultado de calcular las medidas de centralidad para determinar la importancia de los autores en la red. En ambos casos, nos enfocamos en cuatro escenarios seleccionados, los cuales corresponden a la red original y aquellas redes en las que los autores publicaron un mínimo de cinco, siete y diez artículos en coautoría entre el 2010 y el 2019. Los últimos tres escenarios coinciden con un promedio de - aproximadamente- un artículo publicado cada 24,18 y 12 meses, respectivamente.

\subsection{Visualización y descripción de la estructura conectiva de la red}

La base de datos original consta de 2.401 artículos publicados por 1.526 autores, en 426 revistas indexadas en Scopus entre el 2010 y el 2019, bajo la categoría de economía, econometría y finanzas. Del total de publicaciones, el $22.9 \%$ (549 artículos) fue publicado por un solo autor; el $31.1 \%$ (755) fue publicado por dos autores; el 29.2\% (701) fue publicado por tres autores; el $9.4 \%$ (226) fue publicado por cuatro autores, y el 7.1\% (170) fue publicado por cinco o más autores.

Como es usual en la literatura (Newman, 2004), el número de artículos publicados por autor presenta una distribución con una cola derecha particularmente ancha. La figura 1 presenta la distribución del número de artículos por autor en doble escala logarítmica. ${ }^{17}$ La pendiente que describe la distribución (i.e., $\gamma \sim-2,6$ ) corresponde con lo esperado para redes de tipo libre de

17 Es usual utilizar un histograma en doble escala logarítmica para examinar si la distribución se aproxima a una línea recta, donde la pendiente de dicha recta corresponde al exponente de ley de potencia. Sin embargo, como se documenta en Clauset et al. (2009), este método presenta algunos inconvenientes. 
escala (scale-free) antes descritas; esa pendiente muestra que el número de artículos por autor decae muy lentamente, con unos pocos autores produciendo un gran número de artículos.

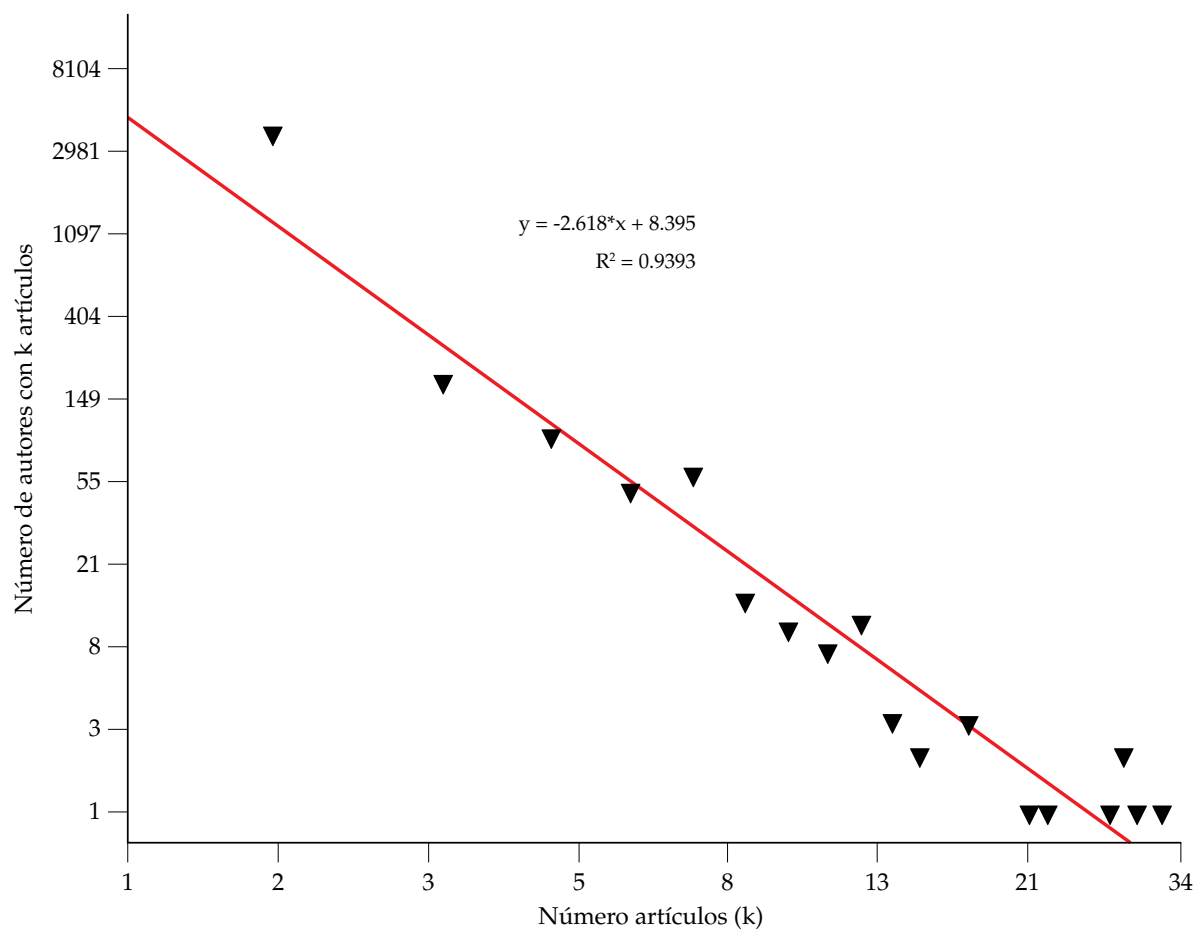

Figura 1. Distribución del número de artículos por autor, en doble escala logarítmica

Fuente: elaboración propia.

La base de datos de coautorías (i.e., sin autores individuales) consta de 1.852 artículos, publicados por 1.208 autores. La figura 2 presenta la visualización de la red que resulta de la coautoría entre esos autores. En este caso, cada autor es representado por un nodo y, las relaciones de coautoría son representadas por las conexiones entre ellos. En esa figura todos los nodos tienen igual tamaño y no se considera la intensidad de las relaciones.

La visualización revela que existen algunas zonas particularmente interconectadas, donde se localizan algunos autores con un número considerable de relaciones de coautoría. En el resto de la red se aprecia que las conexiones son menos numerosas. Esto sugiere que las conexiones en la red siguen algún tipo de proceso que resulta en una estructura jerárquica, en la que existen unos cuantos autores que lideran las coautorías y dan forma a la red. 


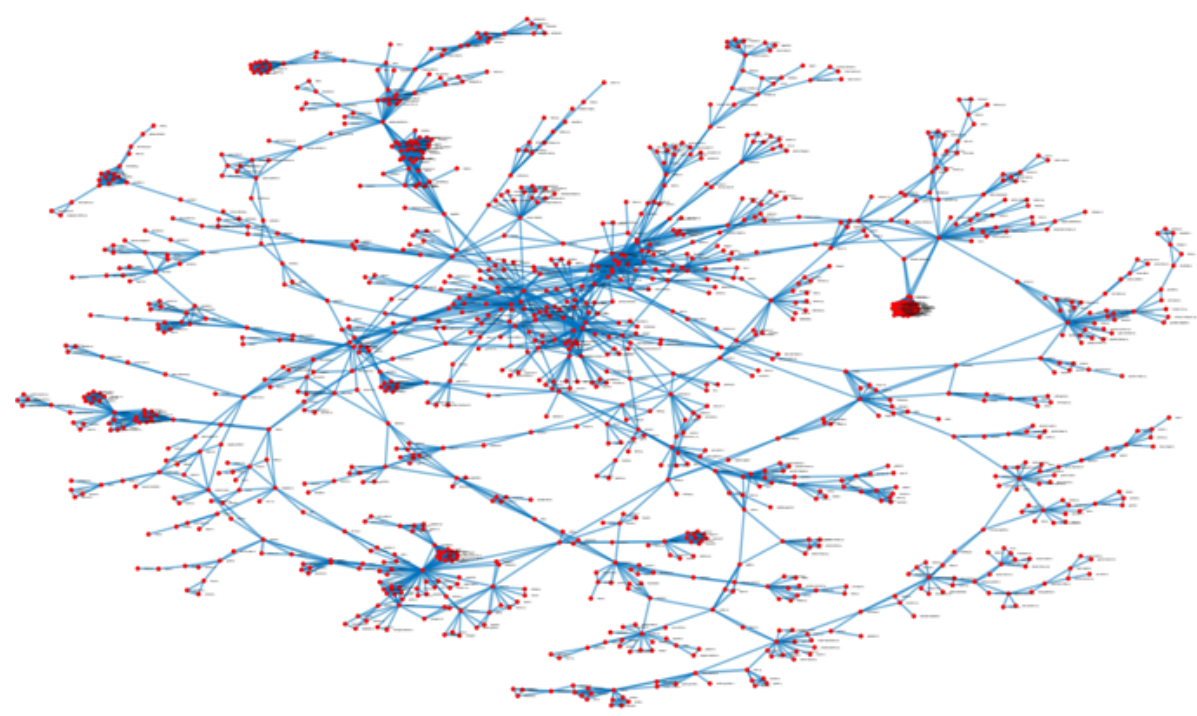

Figura 2. Red de coautorías

Nota: los nodos representan a los autores y las conexiones entre los nodos a las relaciones de coautoría. Todos los nodos tienen igual tamaño y no se considera la intensidad de las relaciones. En este enlace puede visualizar la imagen en alta resolución con los nombres de los autores: https://1drv.ms/u/s!Aj8h_OzuAk t5hsw9fI27z2oztS2uSA?e=GIVHKF

Fuente: elaboración propia.

Al igual que con la distribución de los artículos por autores, la de colaboradores por autor (figura 3) presenta una cola derecha particularmente ancha, lo cual muestra que la mayoría de los autores tienen pocos coautores y que unos pocos autores tienen un gran número de coautores. ${ }^{18}$ La constante aparición de nuevos investigadores y la mayor probabilidad de que estos y otros ya existentes publiquen con aquellos autores con más coautores, ${ }^{19}$ puede explicar la aparición de este tipo de distribución (Barabási et al., 2002; Cárdenas-Tapia, 2021). Esto coincide con el proceso característico de

18 En la figura 3 se aprecia que existe una anomalía en el penúltimo rango - al final de la cola de la distribución-. Corresponde a que tener 48 coautores es tan frecuente como tener 13 coautores. Esta anomalía resulta de cuatro documentos que fueron publicados por más de 45 autores (cada uno). Al excluir este rango, la pendiente es $\gamma \sim-2.09$, con $R^{2}=0.95$, lo cual es consistente con una red libre de escala; no obstante, la pendiente estimada antes de excluir este rango $(\gamma \sim-1.77)$ se aproxima al rango inferior esperado para una red libre de escala.

19 Esta mayor probabilidad puede provenir - entre otros- de la preferencia de los nuevos investigadores por colaborar con autores populares e influyentes en la red, así como de la mayor disponibilidad de recursos (e.g., ideas, datos, financiación) de estos últimos. 
las redes libre de escala (Barabási \& Albert, 1999), el cual se basa en una red cuya evolución está determinada por un proceso de conexión preferencial (i.e., preferential attachment).

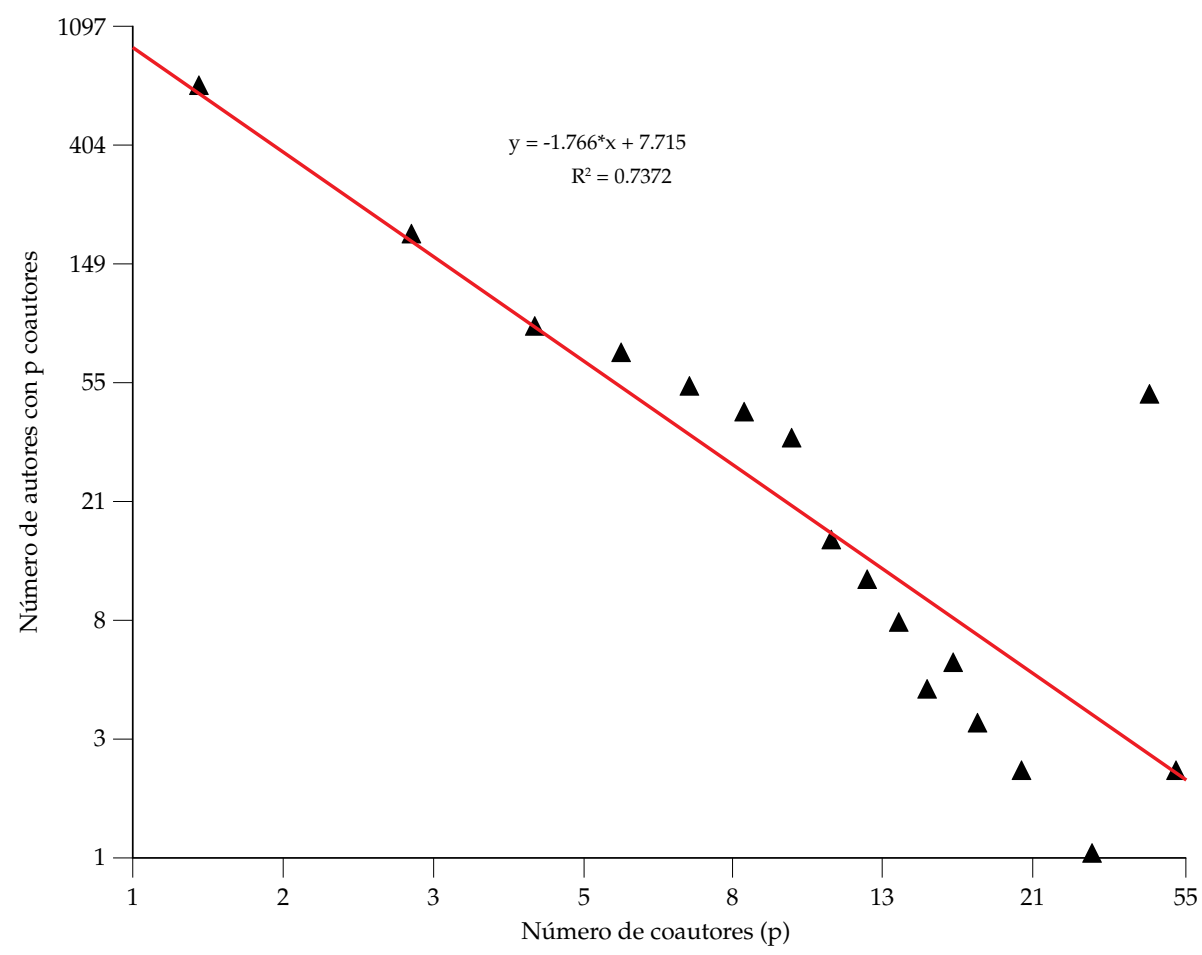

Figura 3. Distribución del número de coautores por autor, en doble escala logarítmica

Fuente: elaboración propia.

Es usual encontrar que las redes de coautoría estén conformadas por componentes que no se interconectan entre sí (Newman, 2004; Liu et al., 2005; Yan \& Ding, 2009; Cárdenas-Tapia, 2021). En estos casos existe un gran componente que agrupa la mayor cantidad de nodos y conexiones en la red, en torno al cual existen - desconectados-otros componentes de menor tamaño. Esta separación por componentes sugiere que existen diferentes áreas del conocimiento que no se relacionan entre sí. En la figura 2 se aprecia que hay un único componente, donde todos los nodos están interconectados directa o indirectamente. A ese respecto, este caso de red de coautoría difiere de lo documentado en la literatura. Según Kumar (2015) y Fatt et al. (2010), esto puede interpretarse como evidencia del buen flujo de conocimiento entre las áreas que componen el campo de economía. Para Newman (2004), 
es un hallazgo positivo: no existe evidencia de aislamiento intelectual en esta red de coautoría.

Si bien es posible estudiar la estructura conectiva de la red de coautorías en la figura 2, esta contiene coautores que han contribuido de manera esporádica o casual a la literatura durante los diez años de la muestra. Lo anterior, se corrobora en la distribución del número de artículos por autor (figura 1) y en la distribución del número de coautores por autor (figura 3). Entonces, con el fin de descartar una gran cantidad de coautorías ocasionales, se seleccionaron tres escenarios.

En el primer escenario se descartó a cualquier autor que no haya conseguido publicar conjuntamente cinco o más artículos en el periodo de diez años de la muestra. En el segundo y tercer escenario, se descartó a cualquier autor que no haya conseguido más de siete y diez artículos en coautoría. Estos tres escenarios corresponden con un promedio de - aproximadamente- un artículo en coautoría cada 24, 18 y 12 meses, respectivamente.

Al descartar a cualquier autor que no haya conseguido publicar conjuntamente cinco o más artículos entre el 2010 y el 2019, se obtuvo una red de 81 coautores. ${ }^{20}$ La red que resulta de ese primer escenario se presenta en la figura 4. De aquí en adelante, cada autor es representado por un nodo cuyo tamaño corresponde al número de artículos publicados -individual o conjuntamente- El color y forma de los nodos revela el género de los coautores: los nodos negros (en forma de círculo) corresponden a autores de género femenino y los rojos (en forma de rombo) corresponden a autores de género masculino. Las relaciones de coautoría son representadas por las conexiones entre los nodos, donde el grosor de las conexiones representa la intensidad (i.e., número de coautorías) entre ellos. Cada nodo está acompañado de los apellidos e iniciales del autor, junto con dos letras (entre corchetes) que identifican su afiliación, así: Banco de la República [BR], Universidad del Rosario [UR], Universidad de los Andes [UA], Universidad de la Sabana [US], ICESI [IC], Universidad del Norte [uo], Universidad de Antioquia [uQ], Universidad del Valle [uv], otros locales [ot] y extranjeros [ex]. ${ }^{21}$

20 De los 1.208 autores de la base de datos original, 162 publicaron cinco o más artículos en coautoría. Sin embargo, solo 81 autores publicaron cinco o más artículos con al menos un coautor, que a su vez publicó cinco o más artículos en coautoría; el resto no aparece conectado a la red. En ese sentido, los escenarios son particularmente exigentes: para que un autor permanezca en la red debe haber publicado con otros que también cumplen con el mínimo de artículos en coautoría.

21 Se creó una categoría para cada afiliación con más de dos autores. Pese a tener su propia categoría, la Universidad Javeriana y la Universidad Nacional no aparecen en los tres escenarios seleccionados. Dentro de la categoría otros locales [ot] se encuentran 


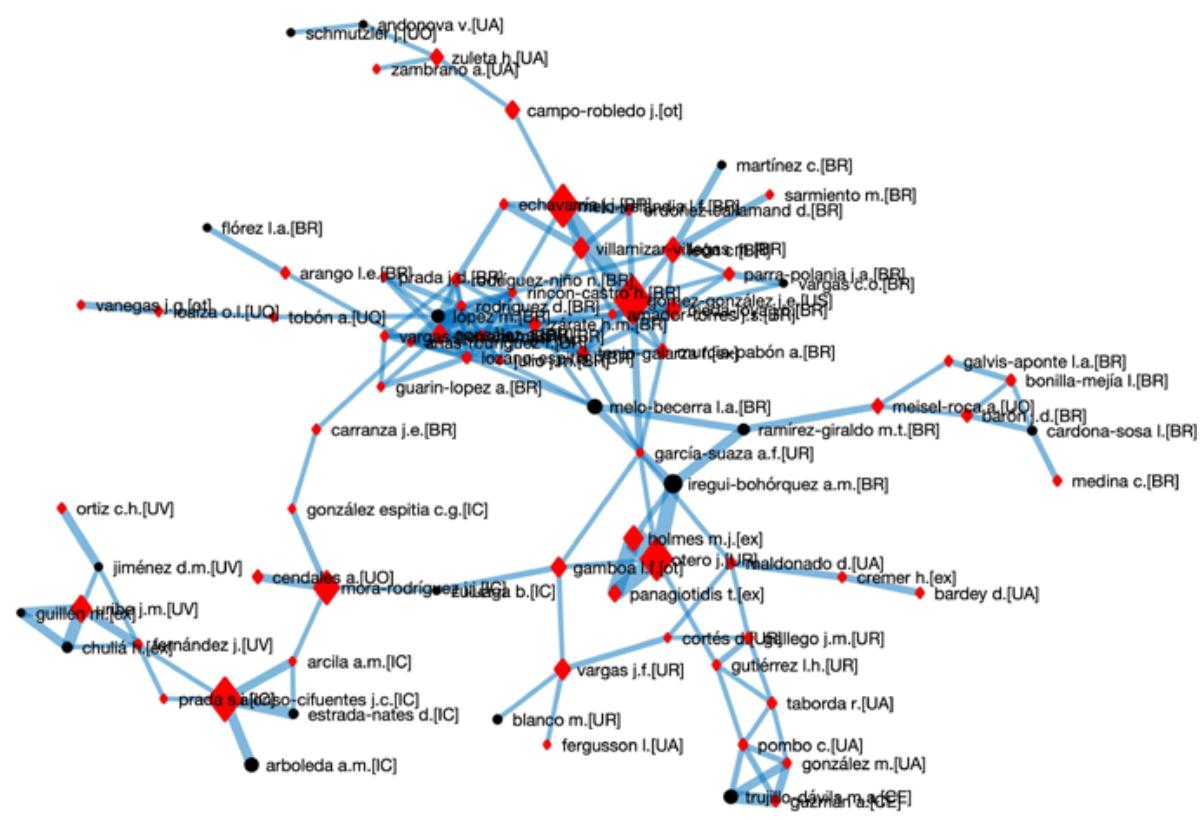

Figura 4. Red de coautorías. Autores con mínimo cinco artículos en coautoría

Nota: los nodos representan a los coautores y las conexiones entre los nodos a las relaciones de coautoría. El tamaño de los nodos representa el total de artículos publicados (individualmente o en coautoría). Los nodos negros (en forma de círculo) corresponden a autores de género femenino y los rojos (en forma de rombo) corresponden a autores de género masculino. En este enlace puede visualizar la imagen en alta resolución: https://1drv.ms/u/s!Aj8h_OzuAkt5hsVqJLburedD_Ii5kA?e=uxeVX3

Fuente: elaboración propia.

Al igual que en la figura 2, es evidente que existen algunas zonas particularmente interconectadas, donde se localizan algunos autores que parecen mantener un número considerable e intenso de relaciones de coautoría entre ellos, mientras que en el resto de la red se aprecia que las conexiones parecen menos numerosas e intensas. Tal como lo reporta Larrosa (2019), es fácil distinguir que las coautorías tienden a suceder entre autores de la misma afiliación. También es evidente que son pocos los nodos correspondientes a coautoras de género femenino, y que existe cierta cercanía entre ellos. Como se amplía en la figura 5, existe una zona particularmente interconectada en la

el Centro Internacional de Agricultura Tropical, el Departamento Nacional de Planeación, Neuroeconomix, la Superintendencia de Industria y Comercio, el Tecnológico de Antioquia Institución Universitaria, la Universidad Católica, la Universidad Industrial de Santander, la Universidad Jorge Tadeo Lozano, la Universidad de la Salle y la Universidad Autónoma de Occidente. 
figura 4. En esta zona es preponderante la afiliación al Banco de la República. ${ }^{22}$ Son los coautores afiliados al Banco de la República quienes consiguen que exista un único componente en la red.

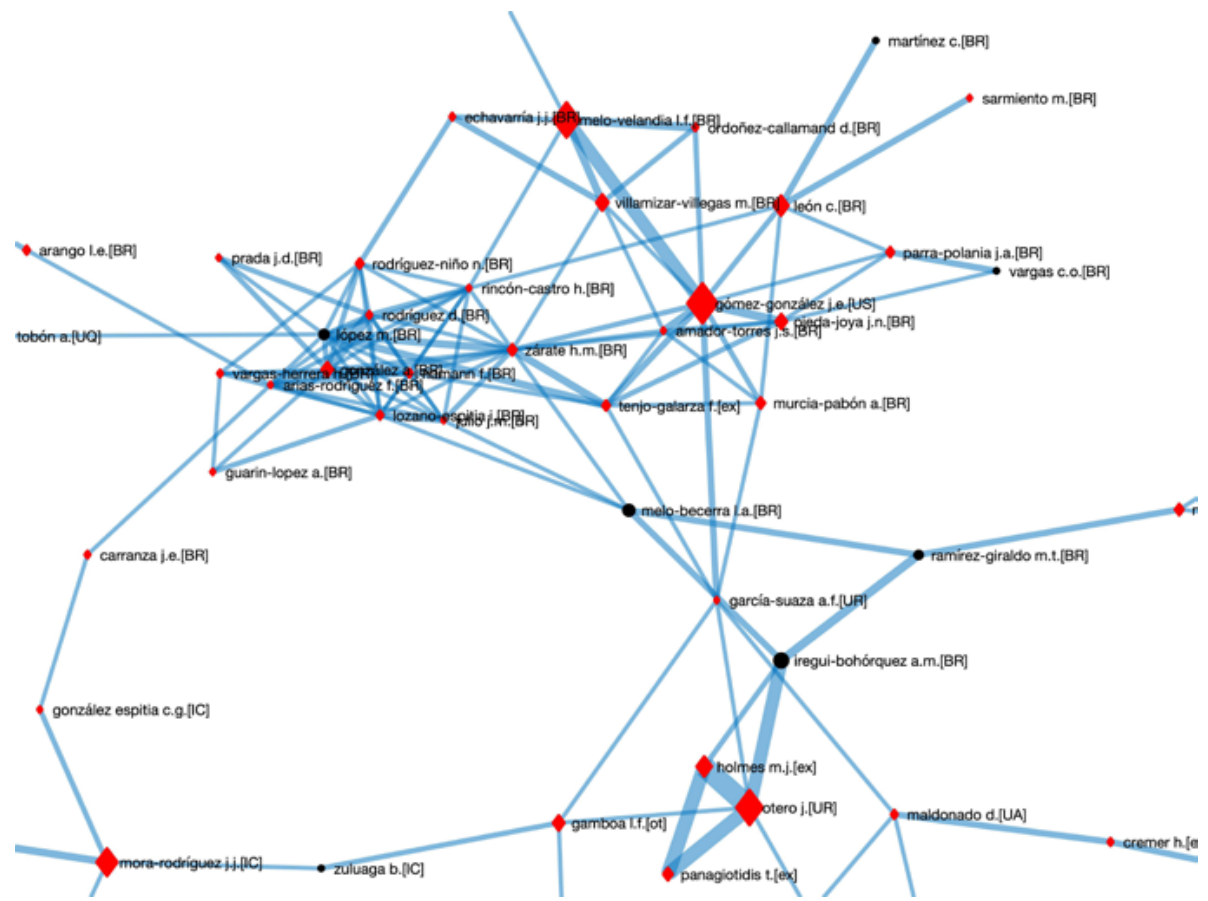

Figura 5. Red de coautorías (acercamiento). Autores con mínimo cinco artículos en coautoría

Nota: los nodos representan a los coautores y las conexiones entre los nodos a las relaciones de coautoría. El tamaño de los nodos representa el total de artículos publicados (individualmente o en coautoría). Los nodos negros (en forma de círculo) corresponden a autores de género femenino y los rojos (en forma de rombo) corresponden a autores de género masculino. En este enlace puede visualizar la imagen en alta resolución: https://1drv.ms/u/s!Aj8h_OzuAkt5hsVrPgnMy6F3GAgofQ?e=aJyav4

Fuente: elaboración propia.

En el segundo escenario (figura 6), al descartar a cualquier autor que no haya conseguido publicar conjuntamente siete o más artículos entre el 2010 y el 2019, se obtiene una red de 28 coautores; de esos 28 coautores, 21 están o estuvieron afiliados al Banco de la República en el periodo de estudio. Persiste una zona particularmente interconectada, en la cual la afiliación al

22 Dos nodos particularmente interconectados en la figura 4 y 5 (Tenjo-Galarza F. [ex] y Gómez-González J.E. [us]), estuvieron afiliados al Banco de la República al inicio del periodo 2010-2019. 
Banco de la República es preponderante. De igual manera, pese a que se ha descartado un gran número de coautores, permanece un único componente en la red; se observa que la colaboración entre tres autoras del Banco de la República (Iregui-Bohórquez A.M. [BR], Melo-Becerra L.A. [BR] y RamírezGiraldo M.T. [BR]) es la que permite que subsista este gran único componente en la red. Por intensidad en las relaciones de coautoría se destaca Otero J. [UR].

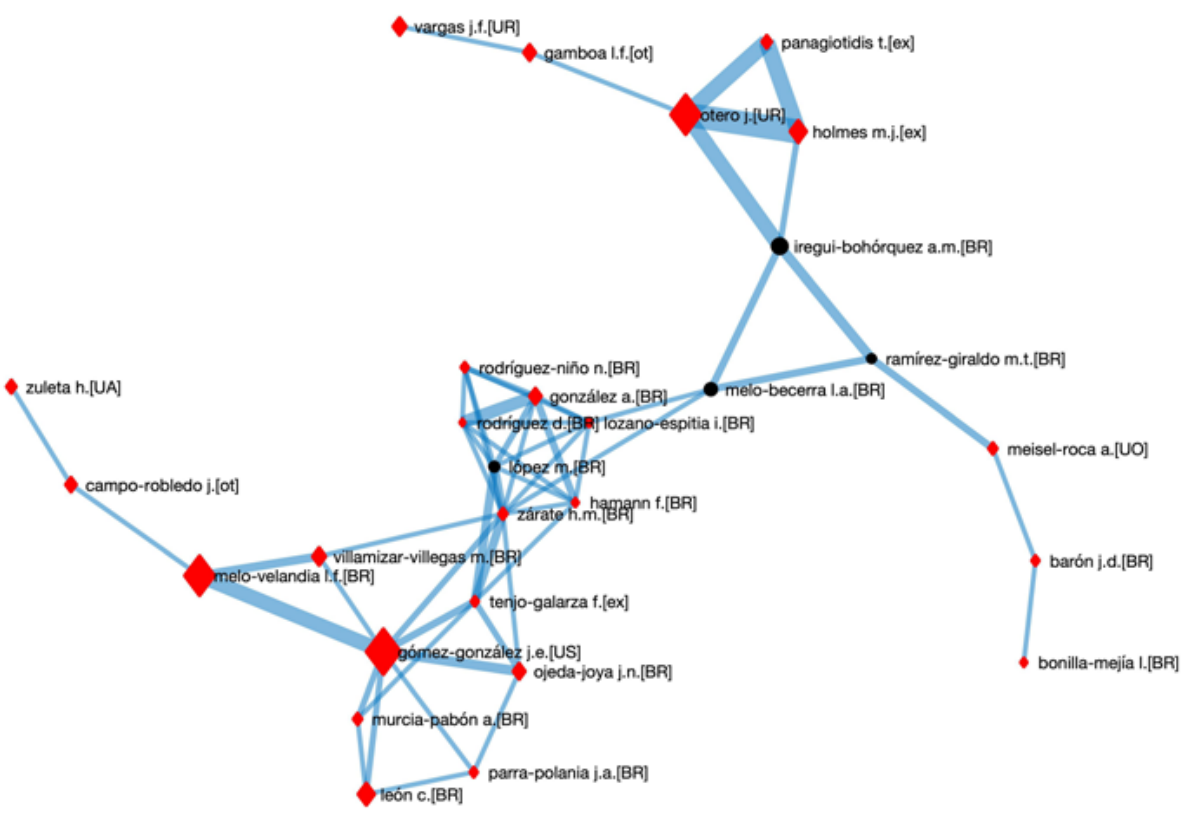

Figura 6. Red de coautorías. Autores con mínimo siete artículos en coautoría

Nota: los nodos representan a los coautores y las conexiones entre los nodos a las relaciones de coautoría. El tamaño de los nodos representa el total de artículos publicados (individualmente o en coautoría). Los nodos negros (en forma de círculo) corresponden a autores de género femenino y los rojos (en forma de rombo) corresponden a autores de género masculino. En este enlace puede visualizar la imagen en alta resolución: https://1drv.ms/u/s!Aj8h_OzuAkt5hsVt2trljnHIWUiLtA?e=9hyroF

Fuente: elaboración propia.

Por último, al descartar a cualquier autor que no haya conseguido publicar conjuntamente diez o más artículos entre el 2010 y el 2019, se obtiene una red de siete coautores (figura 7). Persiste la preponderancia de los autores actual y previamente afiliados al Banco de la República, así como la existencia de un solo componente en la red. No existen coautores de género femenino en esta red. Se destaca la intensa relación de coautoría entre Gómez-González J.E. [Us] y Melo-Velandia L.F. [BR]. 


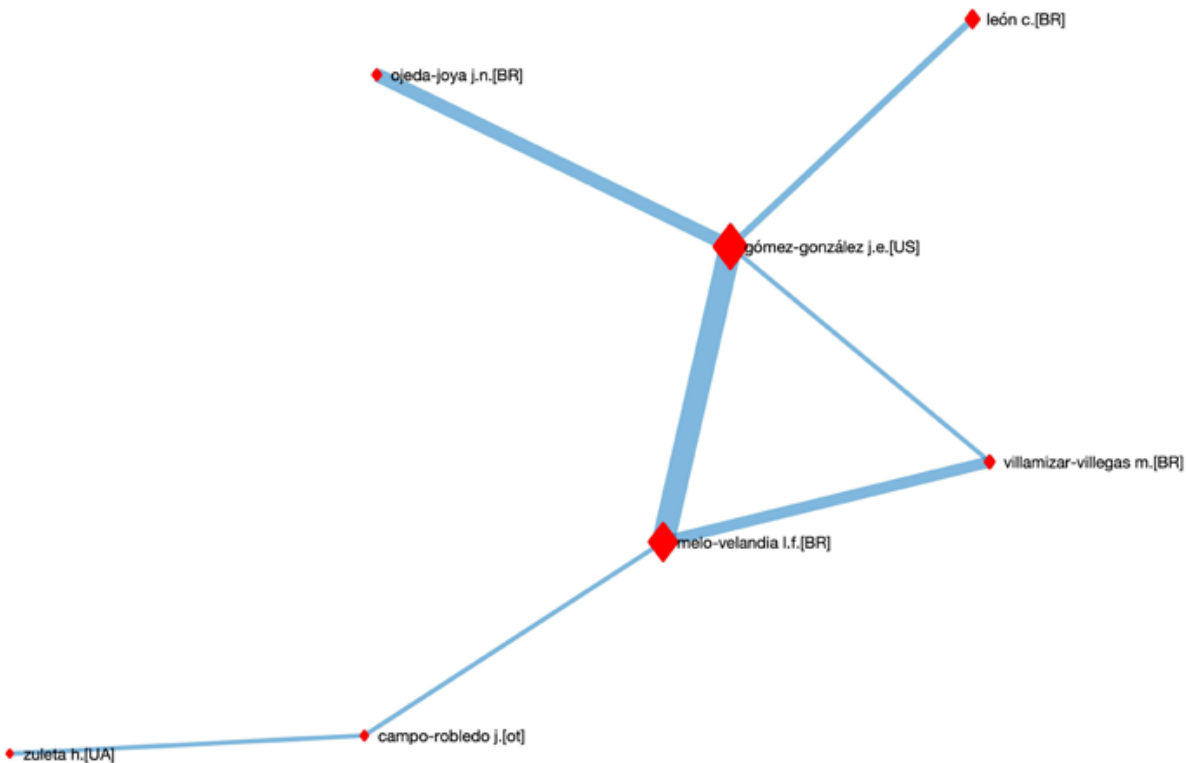

Figura 7. Red de coautorías. Autores con mínimo diez artículos en coautoría

Nota: los nodos representan a los coautores y las conexiones entre los nodos a las relaciones de coautoría. El tamaño de los nodos representa el total de artículos publicados (individualmente o en coautoría). Los nodos negros (en forma de círculo) corresponden a autores de género femenino y los rojos (en forma de rombos) corresponden a autores de género masculino. En este enlace puede visualizar la imagen en alta resolución: https://1drv.ms/u/s!Aj8h_OzuAkt5hsVpg_hqFwVhv8dC9g?e=V0rspB

Fuente: elaboración propia.

Para la red de coautoría original y para las redes de estos tres escenarios, se calcularon las estadísticas básicas descritas en la sección 4.a. Estas estadísticas se presentan en la tabla 1.

Tabla 1. Estadísticas básicas de las redes

\begin{tabular}{|c|c|c|c|c|c|}
\hline \multicolumn{2}{|l|}{ Estadística } & Original a $^{\text {a }}$ & Escenario $1^{\mathrm{b}}$ & Escenario $2^{c}$ & Escenario $3^{\mathrm{d}}$ \\
\hline \multicolumn{2}{|l|}{$\begin{array}{l}\text { Número de coautores en } \\
\text { la red }\end{array}$} & 1.208 & 81 & 28 & 7 \\
\hline \multirow[t]{3}{*}{ Densidad $(\times 100)$} & & 0.56 & 5.22 & 14.81 & 33.33 \\
\hline & Media & 6.77 & 4.17 & 4.00 & 2.00 \\
\hline & $\begin{array}{l}\text { Desviación } \\
\text { estándar }\end{array}$ & 9.83 & 3.17 & 2.39 & 1.16 \\
\hline \multirow[t]{3}{*}{$\begin{array}{l}\text { Distribución de las rela- } \\
\text { ciones de coautoría }\end{array}$} & Sesgo & 3.24 & 1.43 & 1.01 & 0.70 \\
\hline & Curtosis & 13.55 & 4.48 & 3.79 & 2.19 \\
\hline & $\begin{array}{l}\text { Coeficiente ley } \\
\text { de potencia }\end{array}$ & -2.02 & NA & NA & NA \\
\hline
\end{tabular}




\begin{tabular}{lcccc}
\hline \multicolumn{1}{c}{ Estadística } & Original $^{\mathrm{a}}$ & Escenario 1 $^{\mathrm{b}}$ & Escenario 2 $^{\mathrm{c}}$ & Escenario 3 $^{\mathrm{d}}$ \\
\hline Distancia promedio & 8.74 & 5.28 & 3.53 & 2.05 \\
Mayor distancia & 22.00 & 14.00 & 9.00 & 4.00 \\
Transitividad $(\times 100)$ & 66.04 & 26.43 & 31.13 & 11.11 \\
Asortatividad $(\times 100)$ & 87.51 & 54.43 & 29.63 & -49.18 \\
\hline
\end{tabular}

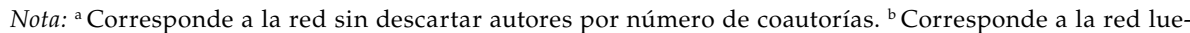

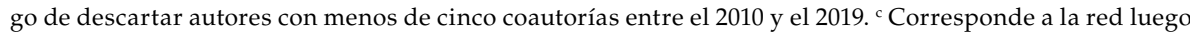
de descartar autores con menos de siete coautorías entre el 2010 y el 2019. d Corresponde a la red luego de descartar autores con menos de diez coautorías entre el 2010 y el 2019. e Por requerir una gran cantidad de datos para su estimación, solo está disponible en la red original. Se estima con el método de máxima verosimilitud de Clauset et al. (2009).

Fuente: elaboración propia.

En general, las estadísticas básicas de las redes coinciden con lo reportado en la literatura respecto a redes de coautoría. La red original (i.e., sin descartar autores por número de coautorías) es de muy baja densidad, los autores colaboran -en promedio- con cerca de siete de sus pares (de entre 1.207 posibles), y las coautorías observadas representan menos del 1\% de las coautorías posibles. La distribución de las relaciones de coautoría es sesgada, con una cola derecha particularmente ancha que decae muy lentamente, cuyo coeficiente de ley de potencia coincide con lo esperado para redes libres de escala. ${ }^{23}$ Pese al gran número de participantes (1.208), estos se encuentran a una distancia promedio de 8.74 relaciones de coautoría, con un máximo de 22. Esa distancia promedio se acerca a lo esperado para redes de tipo mundo pequeño, en la cual la distancia promedio esperada es del orden de $\ln (1.208) \sim 7$. La transitividad es alta $(66.04 \%)$, lo cual es compatible con la gran incidencia de artículos con más de dos coautores y grupos de colaboración en torno a un autor. Igualmente, la alta asortatividad (87.51 \%) muestra que existe una tendencia a que los autores colaboren con otros con similar número de coautores; es decir, autores con muchas relaciones de colaboración tienden a publicar juntos.

23 Determinar si una red es libre de escala es difícil, por cuanto es una propiedad asintótica (i.e., que requiere una red de gran tamaño) que puede estar limitada a la cola de la distribución. En nuestro caso hay tres resultados que indican que es muy posible que así sea: (i) la estimación por mínimos cuadrados ordinarios sobre toda la distribución (en la figura 1 y 3); (ii) la estimación por máxima verosimilitud (Clauset et al., 2009) sobre la cola de la distribución (en la tabla 1), y (iii) la evidencia visual de las distribuciones. En todo caso, sea libre de escala o no, lo importante en este caso es señalar que la distribución de las relaciones de coautoría está particularmente concentrada en unos pocos autores, quienes cumplen una función conectiva determinante en la producción de artículos en colaboración. 
Algunas de estas propiedades de la estructura conectiva de la red persisten al descartar autores en los escenarios elegidos. Es el caso de la transitividad, la baja distancia promedio entre autores (i.e., del orden de $\ln (N))$ y la asortatividad positiva (excepto en el tercer escenario). Otras propiedades varían de manera esperada por la disminución en el número de coautores menos conectados, lo cual resulta en el aumento de la densidad, la reducción en la heterogeneidad, en la distribución de las relaciones de coautoría y en la asortatividad - que se torna negativa en el tercer escenario-. Aunque en general, la estructura conectiva corresponde con lo esperado según la literatura sobre redes de coautoría.

\subsection{La importancia de los autores en la red}

La tabla 2 presenta el resultado de calcular las cinco medidas de centralidad para la red de coautorías del primer escenario seleccionado. ${ }^{24}$ En este escenario existen 81 autores que publicaron en coautoría como mínimo -en promedio- un artículo cada 24 meses entre el 2010 y el 2019. Se presenta el resultado para los primeros 25 autores, según cada medida de centralidad.

La mayoría de los autores en los primeros puestos de la clasificación tienen como afiliación al Banco de la República [BR], seguidos por aquellos afiliados a la Universidad del Rosario [UR], ICESI [IC] y algunos extranjeros [ex]. Resalta que el orden de los autores difiere para cada medida de centralidad, lo cual corresponde a la diferencia conceptual de cada medida, que captura la importancia de los autores desde diferentes perspectivas. Sin embargo, se destaca que Otero J. [UR] ocupa el primer lugar en dos medidas de centralidad —intensidad y eigencentralidad-, ambas calculadas con base en la matriz $\tilde{A}$. Así mismo, se destaca que dos autores, Zárate H.M. [BR] y Gómez-González J.E. [Us], se ubican en los primeros cinco lugares para tres medidas de centralidad diferentes. García-Suaza A.F. [UR] aparece en el primero y segundo lugar por cercanía e intermediación, respectivamente.

24 Por la evidencia de múltiples autores que aparecen con una gran cantidad de coautorías con pocos artículos publicados (i.e., los autores de aquellos artículos con más de 45 coautores) y, en busca de la importancia de coautores que publican habitualmente, se calculan las medidas de centralidad para los tres últimos escenarios solamente. 


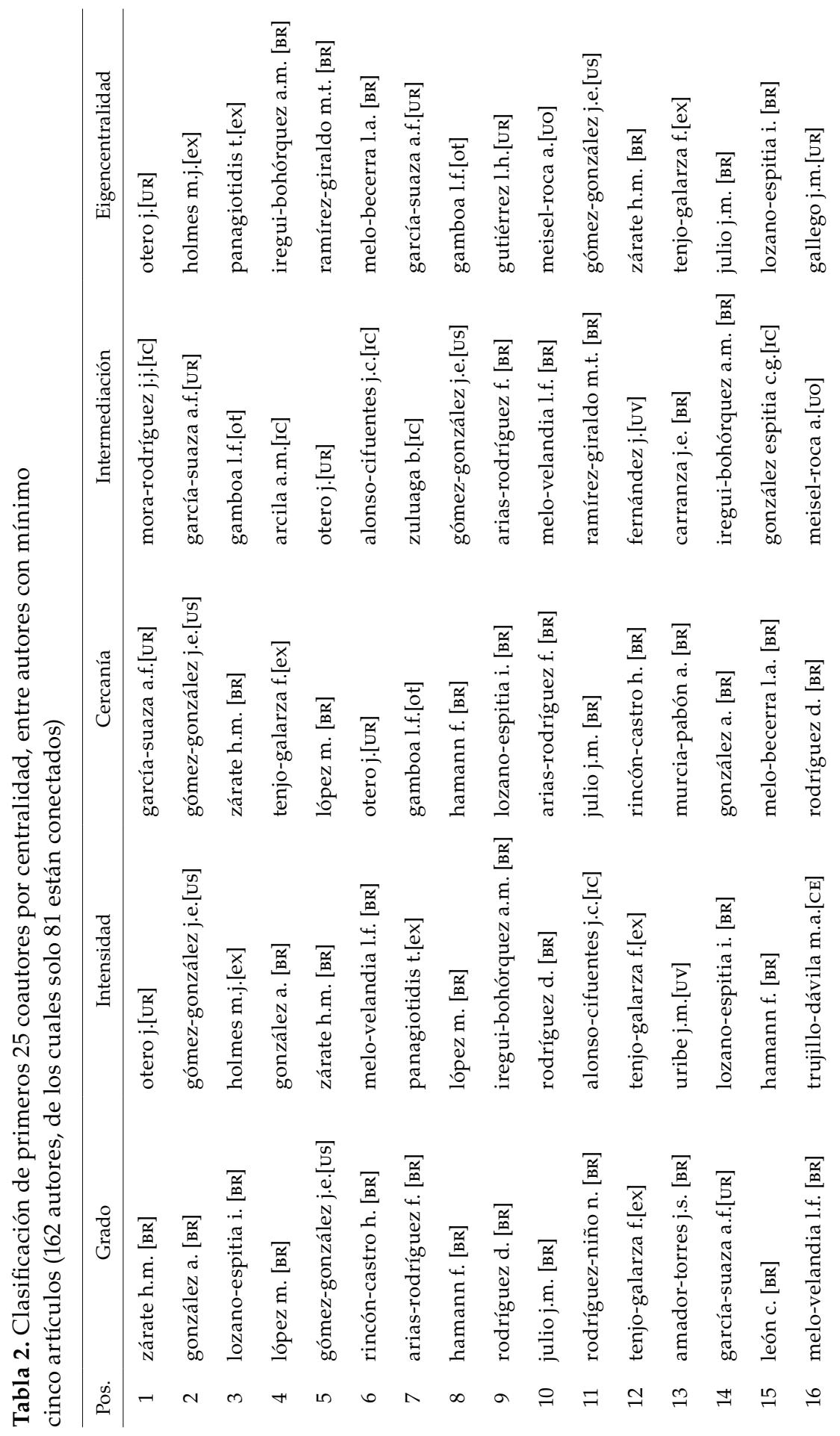




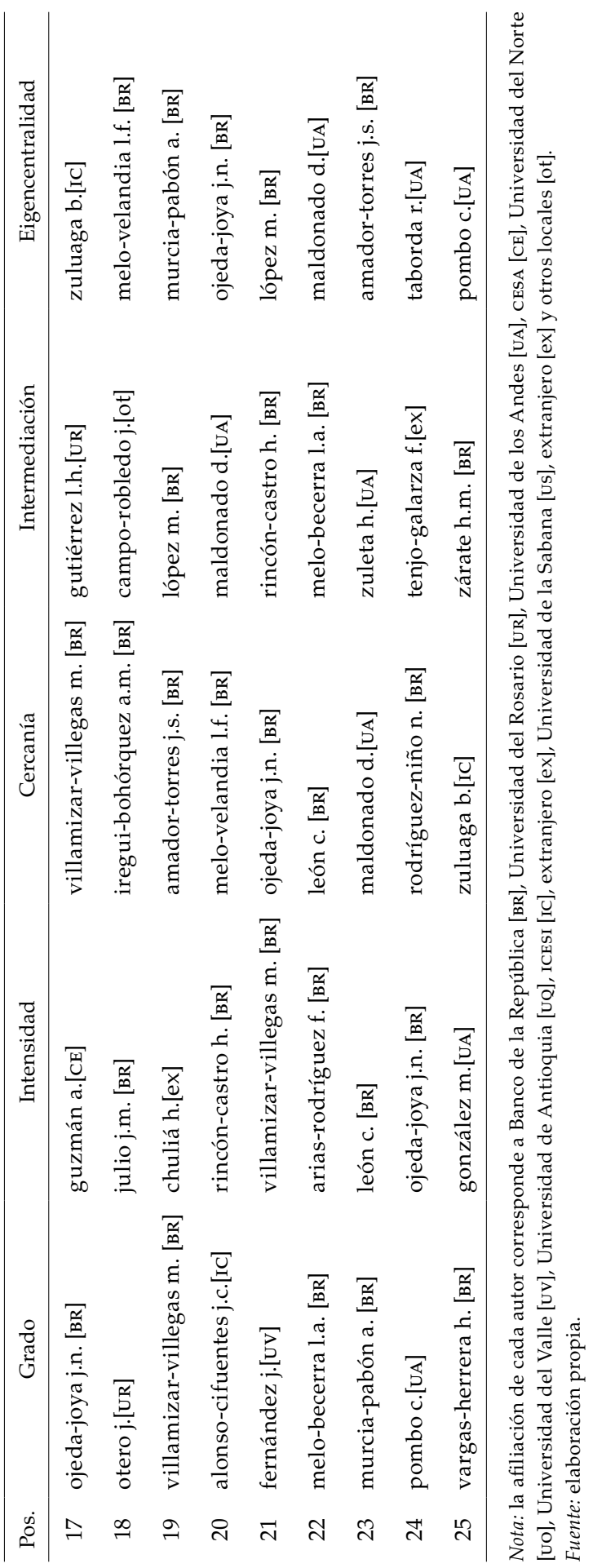


La tabla 3 presenta el resultado de calcular las cinco medidas de centralidad para la red de coautorías del segundo escenario seleccionado. En este escenario existen 28 autores que publicaron en coautoría como mínimo un artículo cada 18 meses (aproximadamente) entre el 2010 y el 2019. Es decir, en la tabla 3 solo aparecen aquellos autores con al menos siete artículos publicados en coautoría. Se presenta el resultado para los primeros 20 autores, según cada medida de centralidad.

Como es de esperar, dado que el segundo escenario es más exigente en cuanto a la contribución de los autores a la producción de artículos, la composición y ordenamiento de los autores en cada medida de centralidad cambia visiblemente. Sin embargo, la mayor importancia de algunos autores (e.g., Otero J. [UR], Gómez-González J.E. [Us], Zárate H.M. [BR]) se mantiene. La preponderancia de los autores afiliados al Banco de la República persiste, mientras que algunas afiliaciones desaparecen en este escenario.

La tabla 4 expone el resultado de calcular las cinco medidas de centralidad para la red de coautorías del tercer escenario seleccionado. La mayor exigencia en cuanto al número de artículos publicados en coautoría (i.e., diez en diez años) resulta en que este escenario solo cuenta con siete autores. Se presenta el resultado para los siete coautores, para cada medida de centralidad.

En este tercer escenario dos autores lideran las cinco medidas de centralidad, Gómez-González J.E. [us] y Melo-Velandia L.F. [BR]. Una vez más, el Banco de la República es la afiliación más recurrente, seguida por la Universidad de la Sabana, la Universidad de los Andes y otros locales.

Finalmente, con el objetivo de tener una clasificación consolidada de los autores, para cada escenario se calculó un índice agregado de centralidad. Este índice utiliza los coeficientes que resultan de utilizar análisis de componentes principales (PCA) sobre las cinco medidas de centralidad calculadas para los autores en cada escenario. ${ }^{25}$ De este modo, se obtiene un índice promedio ponderado de las cinco medidas de centralidad, en las cuales las ponderaciones maximizan el contenido de información de estas. El resultado para cada uno de los tres escenarios vistos se presenta en la tabla 5.

25 PCA es un método no supervisado de reducción de dimensiones, por medio del cual se consigue una representación agnóstica de un conjunto de variables de menor tamaño con la menor pérdida de información posible (Alpaydin, 2014; Mehta et al., 2019). Si $C$ es la matriz de covarianza estimada sobre un conjunto de variables, la descomposición de la forma $C=\Gamma \Lambda \Gamma^{\prime}$, donde $\Gamma$ y $\Lambda$ son la matriz de eigenvectores y eigenvalores, respectivamente, el eigenvector principal (i.e., aquel que coincide con el eigenvalor de mayor magnitud) puede ser utilizado como un vector de ponderaciones o coeficientes para obtener un índice agregado del conjunto de variables. 


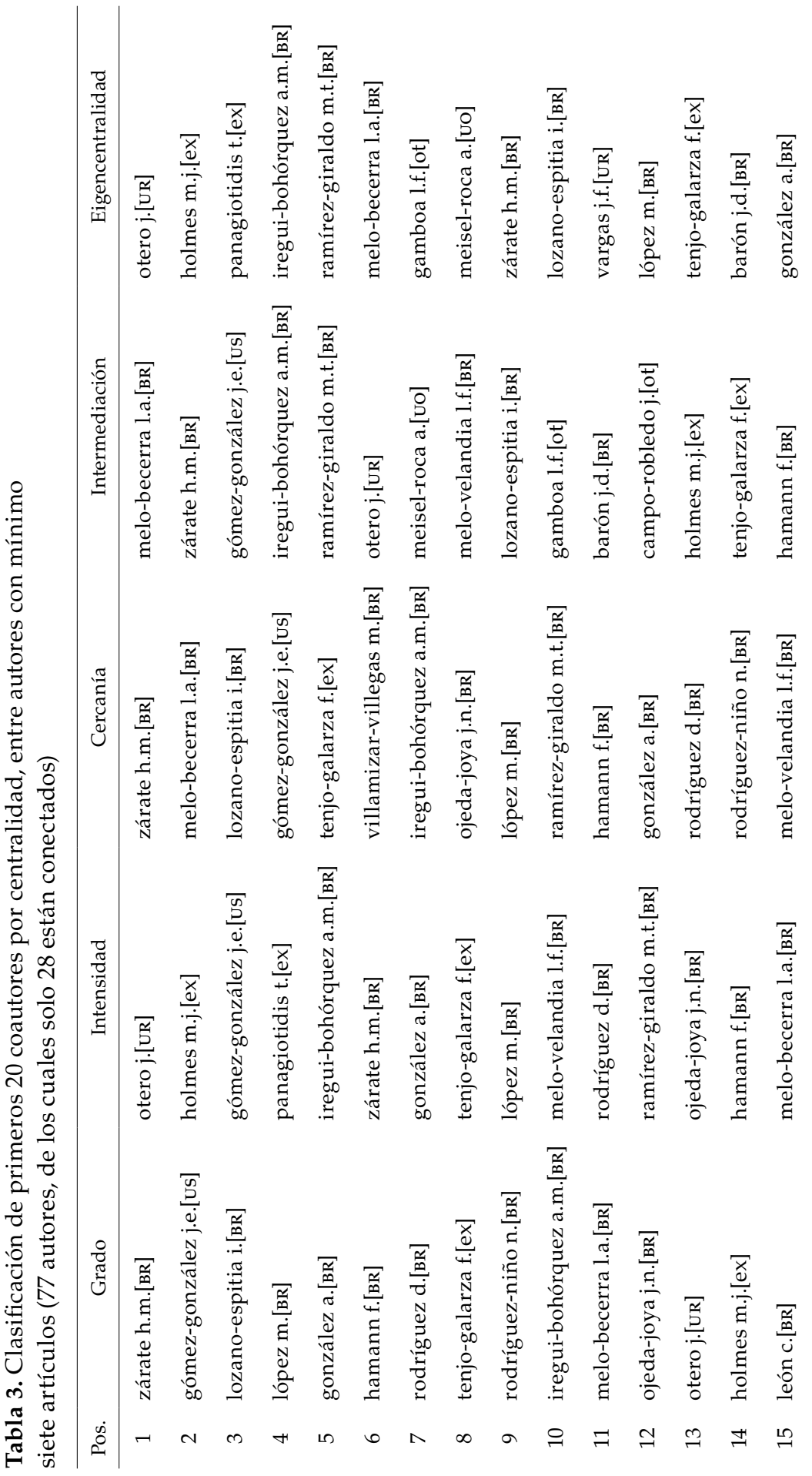




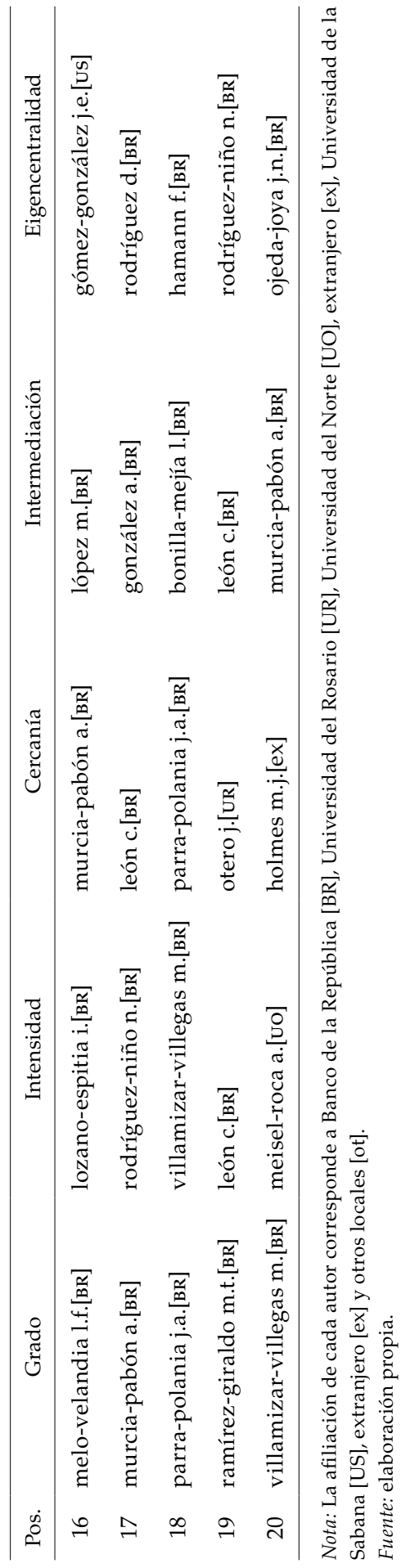

Revista de Economía del Rosario. Vol. 24. No. 2. Julio-Diciembre 2021. 1-39 


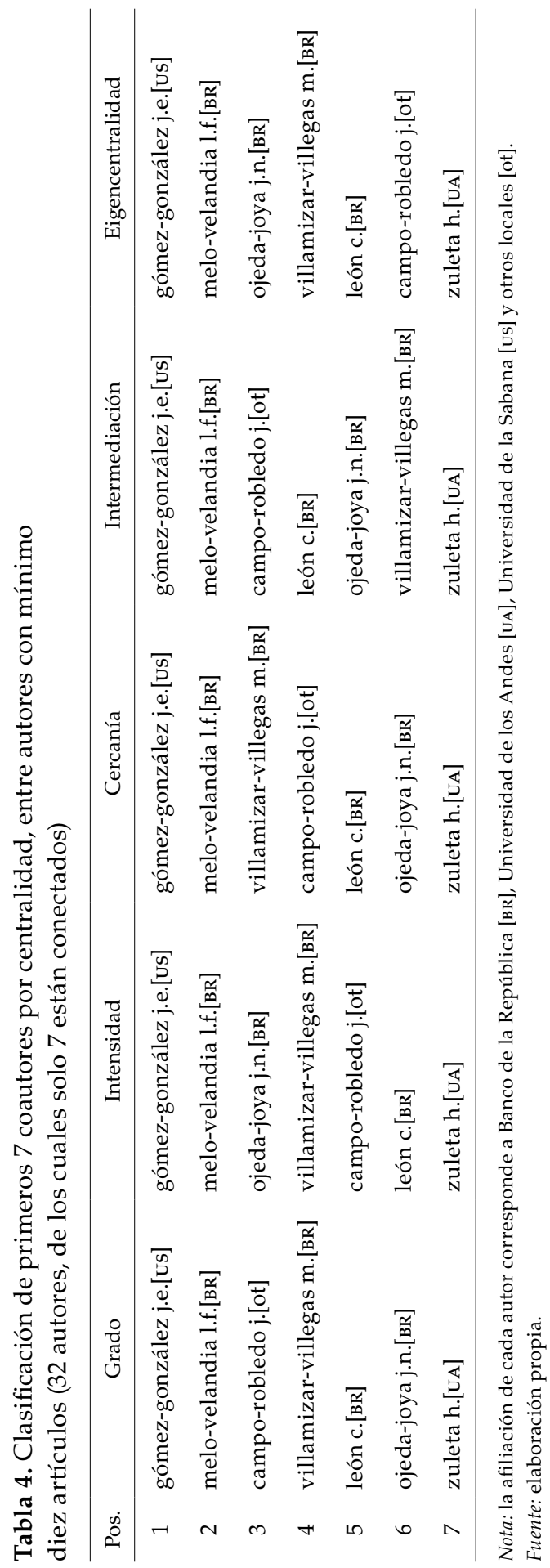


Tabla 5. Clasificación de autores por índice agregado de centralidad para tres escenarios

\begin{tabular}{|c|c|c|c|}
\hline Pos. & Escenario 1 & Escenario 2 & Escenario 3 \\
\hline 1 & otero j.[UR] & zárate h.m.[BR] & gómez-gonzález j.e. [us] \\
\hline 2 & holmes m.j.[ex] & otero j.[UR] & melo-velandia 1.f.[BR] \\
\hline 3 & panagiotidis t.[ex] & gómez-gonzález j.e.[us] & campo-robledo j.[ot] \\
\hline 4 & iregui-bohórquez a.m.[BR] & iregui-bohórquez a.m.[BR] & villamizar-villegas $\mathrm{m}$.[BR] \\
\hline 5 & gómez-gonzález j.e.[us] & melo-becerra l.a.[вR] & ojeda-joya j.n.[BR] \\
\hline 6 & zárate h.m.[BR] & holmes m.j.[ex] & león c.[BR] \\
\hline 7 & lópez m.[BR] & ramírez-giraldo m.t.[BR] & zuleta h. [UA] \\
\hline 8 & garcía-suaza a.f.[UR] & lozano-espitia i.[BR] & \\
\hline 9 & gonzález a.[BR] & panagiotidis t.[ex] & \\
\hline 10 & arias-rodríguez f.[BR] & tenjo-galarza f.[ex] & \\
\hline 11 & melo-velandia l.f.[BR] & lópez m.[BR] & \\
\hline 12 & mora-rodríguez j.j.[IC] & melo-velandia 1.f.[BR] & \\
\hline 13 & gamboa 1.f.[ot] & gonzález a.[BR] & \\
\hline 14 & alonso-cifuentes j.c.[IC] & rodríguez d.[вR] & \\
\hline 15 & rincón-castro h.[BR] & hamann f.[BR] & \\
\hline 16 & tenjo-galarza f.[ex] & meisel-roca a.[uo] & \\
\hline 17 & lozano-espitia i.[BR] & ojeda-joya j.n.[BR] & \\
\hline 18 & ramírez-giraldo m.t.[BR] & rodríguez-niño n.[BR] & \\
\hline 19 & rodríguez d.[BR] & villamizar-villegas m.[BR] & \\
\hline 20 & hamann f.[BR] & gamboa l.f.[ot] & \\
\hline 21 & melo-becerra 1.a.[BR] & barón j.d.[BR] & \\
\hline 22 & arcila a.m.[Ic] & murcia-pabón a.[BR] & \\
\hline 23 & julio j.m.[BR] & campo-robledo j.[ot] & \\
\hline 24 & león c.[BR] & león c.[BR] & \\
\hline 25 & gutiérrez 1.h.[UR] & parra-polania j.a.[BR] & \\
\hline
\end{tabular}

Nota: la afiliación de cada autor corresponde a Banco de la República [BR], Universidad del Rosario [UR], Universidad de los Andes [UA], Universidad del Norte [Uo], ICESI [IC], Universidad de la Sabana [Us], extranjero [ex] y otros locales [ot].

Fuente: elaboración propia. 
En el escenario principal, el primer autor por su centralidad en la red de coautoría es Otero J. [UR], quien ocupa el segundo lugar en el segundo escenario. En el segundo escenario, el primer autor por centralidad es Zárate H.M. [BR]. En el último escenario —el más exigente-, el autor más central en la red de coautoría es Gómez-González J.E. [Us], quien ocupa el quinto y tercer lugar en el primer y segundo escenario, respectivamente. La preponderancia del Banco de la República es evidente en los tres escenarios.

Es importante resaltar que las medidas de centralidad de los autores en la red de coautoría no miden la calidad de los artículos o la influencia de los autores en la red. Desde diferentes perspectivas, las medidas de centralidad seleccionadas reflejan la importancia de los autores como colaboradores en proyectos de investigación.

\section{Comentarios finales}

Al visualizar y describir la estructura conectiva de la red de coautoría de artículos publicados en revistas indexadas en Scopus, del área de economía, entre el 2010 y el 2019, donde alguno de los autores está afiliado a una institución en Colombia, se encontró que esta coincide con aquella reportada para muchos otros tipos de redes, incluyendo redes de coautoría en economía y otras áreas del conocimiento. Es una red de baja densidad y reducida distancia entre autores, en la que la proporción de relaciones transitivas es importante, la distribución de las relaciones es heterogénea y compatible con una red libre de escala y, en la que los autores tienden a relacionarse más con aquellos que le sean semejantes por el número de coautorías. Según Goyal et al. (2006), esta estructura conectiva puede catalogarse como una red de mundo pequeño, lo cual resalta la alta eficiencia de esta red para crear conocimiento a través de la publicación conjunta de artículos y del aprendizaje que supone la colaboración entre autores.

Se destaca la labor del Banco de la República en la estructura conectiva de la red. Es gracias a los coautores afiliados al Banco que diferentes zonas de la red — de diferentes afiliaciones - están conectadas. La contribución a la investigación en el área de economía, econometría y finanzas que realiza el Banco de la República, permite que exista un solo componente en la red y que su estructura sea propicia para la creación de conocimiento a través de la colaboración.

La cuantificación de la centralidad de los autores por su contribución a la producción conjunta de artículos de investigación mostró la importancia de los autores afiliados al Banco de la República y un grupo reducido de universidades. Los resultados también dejan ver una menor contribución de 
coautores de género femenino, lo cual coincide con los hallazgos de Larrosa (2019) y Essers et al. (2020). Las cinco medidas de centralidad implementadas, así como el índice agregado de centralidad, complementan el Top 100 de autores colombianos según RePEc, el cual es publicado periódicamente por el convenio Red de Investigadores de Economía.

De este estudio surgen varias preguntas y posibles líneas de investigación. Primero, como se estudia en Yan y Ding (2009), Uddin et al. (2013), Rath y Wohlrabe (2016), ¿existe alguna relación significativa entre la importancia en la red de coautoría y las medidas de popularidad, visibilidad y reconocimiento de los artículos (i.e., citaciones), revistas (i.e., factor de impacto) y autores (i.e., índices h)? Segundo, como se analiza en Hollis (2001), Łopaciuk-Gonczaryk et al. (2015), Larrosa (2019) y Gosh y Liu (2020), ¿existe alguna relación significativa entre la importancia en la red de coautoría y las características de los autores (e.g., edad, género, nivel de estudios, afiliación, años desde graduación, alma máter)? Tercero, teniendo en cuenta el número de universidades e instituciones de investigación en temas de economía en Colombia, ¿qué explica la evidente concentración de las afiliaciones? Cuarto, ¿cómo ha sido la evolución de la red de coautoría a través del tiempo? Quinto, con base en las palabras clave de los artículos, ¿existe especialización o interdisciplinariedad en la colaboración entre autores en la red? Sexto, ya que la intersección entre economía, física, matemáticas aplicadas y ciencias de la computación es bien conocida (Tabak et al., 2021), no restringir las revistas indexadas al área de economía, econometría y finanzas permitiría estudiar cómo la creación de conocimiento en el área de economía tiene un carácter multidisciplinar. Séptimo, dado que no existen componentes desconectados en la red de coautoría, resulta interesante implementar herramientas de detección de comunidades para estudiar si existen grupos que revelen las diferentes áreas de conocimiento de la red. Finalmente, es interesante examinar si este tipo de estudios puede inducir cambios en el comportamiento de los autores, como sugiere Katz y Martin (1997). Nuestro artículo brinda algunas bases para responder estas preguntas.

\section{Referencias}

Albert, R., \& Barabási, A.-L. (2002). Statistical mechanics of complex networks. Reviews of Modern Physics, 74(1), 47-97. https://doi.org/10.1103/ RevModPhys.74.47

Alpaydin, E. (2014). Introduction to Machine Learning. The MIT Press.

Barabási, A.-L., \& Albert, R. (1999). Emergence of scaling in random networks. Science, 286(5439), 509-512. https://doi.org/10.1126/science.286.5439.509 
Barabási, A.-L., Jeong, H., Néda, Z., Ravasz, E., Schubert, A., \& Vicsek, T. (2002). Evolution of the social network of scientific collaborations. Physica A: Statistical mechanics and its applications, 311(3-4), 590-614. https:// doi.org/10.1016/S0378-4371(02)00736-7

Börner, K., Sanyal, S., \& Vespignani, A. (2007). Network science. Annual Review of Information Science and Technology, 41(1), 537-607. https://doi. org/10.1002/aris.2007.1440410119

Cárdenas-Tapia, M. (2021). Redes de colaboración científica en el Instituto Politécnico Nacional de México. Investigación Administrativa, 50(127), 192-212. https://doi.org/10.35426/iav50n127.06

Clauset, A., Shalizi, C.R., \& Newman, M.E.J. (2009). Power-law distributions in empirical data. SIAM Review, 51(4), 661-703. https://doi. org/10.1137/070710111

Essers, D., Grigoli, F., \& Pugacheva, E. (2020). Network effects and research collaborations (IMF Working Paper n. ${ }^{\circ}$ 144). International Monetary Fund.

Fatt, C.K., Ujum, E.A., \& Ratnavelu, K. (2010). The structure of collaboration in the Journal of Finance. Scientometrics, 85(3), 849-860. https://doi. org/10.1007/s11192-010-0254-0

Gosh, P., \& Liu, Z. (2020). Coauthorship and the gender gap in top economics journal publications. Applied Economic Letters, 27(7), 580-590. https://doi. org/10.1080/13504851.2019.1644420

Goyal, S., Van der Leij, M., \& Moraga-González, J.L. (2006). An emerging small world. Journal of Political Economy, 114(2), 403-412. https://doi. org/10.1086/500990

Hamermesh, D. (2020). Measuring success in economics. En S. Galiani \& U. Panizza (Eds.), Publishing and Measuring Success in Economics. CEPR Press. https://n9.cl/15z65

Hollis, A. (2001). Co-authorship and the output of academic economists. Labour Economics, 8(4), 503-530. https://doi.org/10.1016/S0927-5371(01)00041-0

Hsieh, C.-S., König, M.D., Liu, X., \& Zimmermann, C. (2018). Superstar Economists: Coauthorship Networks and Research Output (IzA Working Papers n. $\left.{ }^{\circ} 11916\right)$. Institute of Labor Economics. https://papers.ssrn.com/sol3/ papers.cfm?abstract_id=3266432

Katz, J.S., \& Martin, B.R. (1997). What is research collaboration?. Research Policy, 26(1), 1-18. https://doi.org/10.1016/S0048-7333(96)00917-1

Kumar, S. (2015). Co-authorship networks: a review of the literature. Aslib Journal of Information Management, 67(1), 55-73. https://doi.org/10.1108/ AJIM-09-2014-0116 
Larrosa, J.C. (2019). Co-authorship networks of Argentine economists. Journal of Economics, Finance and Administrative Science, 24(47), 82-96. https://doi. org/10.1108/JEFAS-06-2018-0062

Liebowitz, S. (2020). Our uneconomic methods of measuring economic research. En S. Galiani \& U. Panizza (Eds.), Publishing and Measuring Success in Economics. CEPR Press.

Liu, X., Bollen, J., Nelson, M.L., \& Van de Sompel, H. (2005). Co-authorship networks in the digital library research community. Information processing $\mathcal{E}$ Management, 41(6), 1462-1480. https://doi.org/10.1016/j.ipm.2005.03.012 Łopaciuk-Gonczaryk, B., Bukowska, G., \& Fałkowski, J. (2015). Teaming up or writing alone - authorship strategies in leading polish economic journals. Argumenta Oeconomica, 2(35), 5-24. https://www.wne.uw.edu. pl/files/2314/2244/2304/WNE_WP146.pdf

Lu, C., Zhang, Y., Ahn, Y-Y., Ding, Y., Zhang, C., \& Ma, D. (2019). Co-contributorship network and division of labor in individual scientific collaborations. Journal of the Association for Information Science and Technolo$g y, 71(10), 1162-1178$. https://doi.org/10.1002/asi.24321

Martín-Martín, A., Orduna-Malea, E., Thelwall, M., \& Delgado López-Cózar, E. (2018). Google Scholar, Web of Science, and Scopus: A systematic comparison of citations in 252 subject categories. Journal of Informetrics, 12(4), 1160-1177. https://doi.org/10.1016/j.joi.2018.09.002

Mehta, P., Bukov, M., Wang, C.-H., Day, A.G.R., Richardson, C., Fisher, C.K., \& Schwab, D.J. (2019). A high-bias, low-variance introduction to Machine Learning for physicists. Physics reports, 810, 1-124. https://doi. org/10.1016/j.physrep.2019.03.001

Melin, G., \& Persson, O. (1996). Studying research collaboration using coauthorships. Scientometrics, 36(3), 363-377. https://doi.org/10.1007/ bf02129600

Newman, M.E.J. (2001). Scientific collaboration networks (I. Network construction and fundamental results). Physical Review E, 64. https://doi. org/10.1103/PhysRevE.64.016131

Newman, M.E.J. (2004). Coauthorship networks and patterns of scientific collaboration. Proceedings of the National Academy of Sciences of the United States of America, 101, 5200-5205. https://doi.org/10.1073/pnas.0307545100 Newman, M.E.J. (2010). Networks: An Introduction. Oxford University Press. O'Hagan, J., \& Kuld, L. (2020). Multi-authored journal articles in economics: Why the spiralling upward trend?. En S. Galiani \& U. Panizza (Eds.), Publishing and Measuring Success in Economics. CEPR Press. 
Rath, K., \& Wohlrabe, K. (2016). Recent trends in co-authorship in economics: evidence from RePEc. Applied Economic Letters, 23(12), 897-902. https:// doi.org/10.1080/13504851.2015.1119783

Tabak, B.M., Silva, T.C., Fiche, M.E., \& Braz, T. (2021). Citation likelihood analysis of the interbank financial networks literature: A machine learning and bibliometric approach. Physica A, 562. https://doi.org/10.1016/j. physa.2020.125363

Uddin, S., Hossain, L., \& Rasmussen, K. (2013). Network effects on scientific collaborations. PLoS One, 8(2). https://doi.org/10.1371/journal. pone. 0057546

Yan, E., \& Ding, Y. (2009). Applying centrality measures to impact analysis: A coauthorship network analysis. Journal of the Association for Information Science and Technology, 71(10), 2107-2118. https://doi.org/10.1002/asi.21128 


\section{Apéndices}

Apéndice A. Estadísticas básicas de las redes y medidas de centralidad ${ }^{26}$

$$
d=\frac{m}{N(N-1)}
$$

Densidad

$$
l=\frac{1}{N} \sum_{i} l_{i}
$$

Distancia promedio entre autores en la red

$$
r=\frac{\sum_{i j}\left(A_{i j}-k_{i} k_{j} / 2 m\right) k_{i} k_{j}}{\sum_{i j}\left(k_{i} \delta_{i j}-k_{i} k_{j} / 2 m\right) k_{i} k_{j}}
$$

Asortatividad

$$
S_{i}=\sum_{j=1}^{N} \tilde{A}_{i j}
$$

Centralidad de intensidad del autor $i$

$$
q_{i}=\frac{1}{l_{i}}
$$

Centralidad de cercanía del autor $i$

$$
l_{i}=\frac{1}{(N-1)} \sum_{j(\neq i)} g_{i j}
$$

Distancia promedio del autor $i$

$$
c=\frac{\text { (número de triángulos en la red) } \times 3}{\text { número de conjuntos con tres vértices }}
$$

Coeficiente de clustering

(i.e., transitividad)

$$
k_{i}=\sum_{j=1}^{N} A_{i j}
$$

Centralidad de grado del autor $i$

$$
b_{i}=\sum_{i \neq j \neq k}^{N} \frac{L_{I k j}}{L_{i j}}
$$

Centralidad de interposición del autor $i$

$$
e_{i}=\Gamma_{i}^{1}\left(\tilde{A}_{j i}\right)
$$

Centralidad de eigenvector del autor $i$ 
Donde:

$A_{i j}$ es la matriz de adyacencia

$\tilde{A}_{j i}$ es la matriz de adyacencia ponderada

$g_{i j}$ es la distancia más corta entre $i$ y $j$

$L_{i j}$ es el número de caminos más cortos entre $i$ y $j$

$L_{i k j}$ es el número de caminos más cortos entre $i$ y $j$ que pasan por $k$

$N$ es el número de elementos o nodos en la red

$m$ es el número de conexiones observadas en la red

$\Gamma_{i}^{1}(X)$ es el elemento $i$ del primer (principal) eigenvector de una matriz $X$

$\delta_{i j} \begin{cases}0 & \text { if } i \neq j \\ 1 & \text { if } i \neq j\end{cases}$ 


\section{Apéndice B. Estadísticas básicas de las redes y medidas de centralidad}

\begin{tabular}{|c|c|c|c|}
\hline Base de datos & Descripción & Requisitos de entrada & Ventajas y desventajas \\
\hline $\begin{array}{l}\text { Web of Science } \\
\text { (Core Collection) }\end{array}$ & $\begin{array}{l}\text { Base de datos } \\
\text { multidisciplinar } \\
\text { de referencias bi- } \\
\text { bliográficas y citas } \\
\text { de publicaciones } \\
\text { revisadas por pa- } \\
\text { res. Acceso a cerca } \\
\text { de } 21.100 \text { piezas de } \\
\text { investigación. }\end{array}$ & $\begin{array}{l}\text { Se indexan revistas } \\
\text { que han superado } \\
\text { requisitos de políti- } \\
\text { cas de publicación, } \\
\text { contenido editorial, } \\
\text { diversidad interna- } \\
\text { cional de autores y } \\
\text { editores y, análisis } \\
\text { de citas. }\end{array}$ & $\begin{array}{l}\text { Ventajas: tiene filtros de } \\
\text { entrada. Permite identificar } \\
\text { la afiliación del documento. } \\
\text { Desventajas: requiere sus- } \\
\text { cripción. }\end{array}$ \\
\hline Scopus & $\begin{array}{l}\text { Es la mayor base } \\
\text { de datos multidis- } \\
\text { ciplinar de citas y } \\
\text { resúmenes biblio- } \\
\text { gráficos de publi- } \\
\text { caciones evaluadas } \\
\text { por pares. Acceso } \\
\text { a cerca de } 39.500 \\
\text { piezas de investi- } \\
\text { gación. }\end{array}$ & $\begin{array}{l}\text { Se indexan revistas } \\
\text { que han superado } \\
\text { requisitos de políti- } \\
\text { cas de publicación, } \\
\text { calidad del conteni- } \\
\text { do, cumplimiento de } \\
\text { la periodicidad de- } \\
\text { clarada y, análisis } \\
\text { de citas. }\end{array}$ & $\begin{array}{l}\text { Ventajas: tiene filtros de } \\
\text { entrada. Permite identificar } \\
\text { la afiliación del documen- } \\
\text { to. A diferencia de Web of } \\
\text { Science-Core Collection, } \\
\text { tiene una mayor cobertu- } \\
\text { ra en Ciencias Sociales y } \\
\text { documentos escritos en } \\
\text { español. } \\
\text { Desventajas: requiere sus- } \\
\text { cripción. }\end{array}$ \\
\hline RePEc & $\begin{array}{l}\text { Repositorio temáti- } \\
\text { co con acceso a más } \\
\text { de } 3 \text { millones de } \\
\text { piezas de investiga- } \\
\text { ción, provenientes } \\
\text { de } 3.500 \text { revistas } \\
\text { y } 5.000 \text { series de } \\
\text { documentos de } \\
\text { trabajo especiali- } \\
\text { zados en el área de } \\
\text { economía. }\end{array}$ & $\begin{array}{l}\text { Admite artículos, do- } \\
\text { cumentos de trabajo, } \\
\text { libros y capítulos de } \\
\text { libro. }\end{array}$ & $\begin{array}{l}\text { Ventajas: gran cantidad } \\
\text { de documentos. De acceso } \\
\text { libre. Genera rankings de } \\
\text { instituciones, series y au- } \\
\text { tores. } \\
\text { Desventajas: la identifica- } \\
\text { ción de la afiliación se hace } \\
\text { a nivel de autor, cuando un } \\
\text { autor cambia de institución } \\
\text { "arrastra" su producción } \\
\text { académica a la nueva afilia- } \\
\text { ción. No se realiza ningún } \\
\text { filtro. }\end{array}$ \\
\hline Google Scholar & $\begin{array}{l}\text { Buscador especia- } \\
\text { lizado en literatura } \\
\text { académica de dife- } \\
\text { rentes disciplinas. }\end{array}$ & $\begin{array}{l}\text { Indexa todo lo que } \\
\text { Google identifica } \\
\text { como documento } \\
\text { académico (e.g., li- } \\
\text { bros, documentos } \\
\text { de trabajo, artículos, } \\
\text { informes, actas de } \\
\text { congresos). }\end{array}$ & $\begin{array}{l}\text { Ventajas: gran cantidad } \\
\text { de documentos. De acceso } \\
\text { libre. } \\
\text { Desventajas: no se realiza } \\
\text { ningún filtro. }\end{array}$ \\
\hline
\end{tabular}

Nota: descripción de las bases de datos disponibles.

Fuente: elaboración propia. 